\title{
Modeling of the Jovian Magnetosphere
}

\author{
I. I. Alexeev and E. S. Belenkaya \\ Institute of Nuclear Physics, Moscow State University, Moscow, 119992, Russia
}

Received: 31 May 2004 - Revised: 27 December 2004 - Accepted: 1 February 2005 - Published: 30 March 2005

\begin{abstract}
This paper presents a global model of the Jovian magnetosphere which is valid not only in the equatorial plane and near the planet, as most of the existing models are, but also at high latitudes and in the outer regions of the magnetosphere. The model includes the Jovian dipole, magnetodisc, and tail current system. The tail currents are combined with the magnetopause closure currents. All inner magnetospheric magnetic field sources are screened by the magnetopause currents. It guarantees a zero normal magnetic field component for the inner magnetospheric field at the whole magnetopause surface. By changing magnetospheric scale (subsolar distance), the model gives a possibility to study the solar wind influence on the magnetospheric structure and auroral activity. A dependence of the magnetospheric size on the solar wind dynamic pressure $p_{s w}$ (proportional to $p_{s w}^{-0.23}$ ) is obtained. It is a stronger dependence than in the case of the Earth's magnetosphere $\left(p_{s w}^{-1 / 6}\right)$. The model of Jupiter's magnetospheric which is presented is a unique one, as it allows one to study the solar wind and interplanetary magnetic field (IMF) effects.
\end{abstract}

Keywords. Magnetospheric physics (Planetary magnetospheres; Plasma convection; Solar wind-magnetosphere interactions)

\section{Introduction}

The presented Jovian magnetospheric model is constructed on the basis of the Alexeev (1986) paraboloid model of the terrestrial magnetospheric magnetic field. In this paper we describe a dynamic Jovian magnetospheric model which is not connected directly with specific spacecraft flyby. It allows one to calculate the magnetospheric response to variations in the solar wind dynamic pressure and magnetic field. For this reason the magnetospheric global current systems are constructed depending on a small number of parameters, each with a clear physical meaning.

Correspondence to: I. I. Alexeev

(alexeev@dec1.npi.msu.su)
In the solar wind the sonic and Alfvén Mach numbers are large and the pressure is dominated by the dynamic pressure. Following Slavin et al. (1985), at the subsolar Jovian magnetopause we can calculate the solar wind pressure $p_{s w J}=0.58 p_{0 J}$, where $p_{0 J}=2 m_{p} n_{s w J} V_{s w}^{2}$ is the dynamic pressure for the perfectly elastic collisions of the solar wind ions with the magnetopause. Here $m_{p}$ is the proton mass, $V_{s w}$ is the solar wind speed, and $n_{s w J}$ is the number of solar wind ions per $\mathrm{cm}^{3}$ at Jupiter's orbit. According to Spreiter et al. (1966) at the Earth's magnetopause, the solar wind pressure is $p_{s w E}=0.88 p_{0 E}$, where $p_{0 E}=2 m_{p} n_{s w E} V_{s w}^{2}$ with $n_{s w E}$ being the number of ions per $\mathrm{cm}^{3}$ at the Earth's orbit. The factors 0.58 and 0.88 are empirical values of the decrease in pressure across the subsolar magnetosheath.

Thus, to calculate the solar wind pressure we must multiply the pressure at the subsolar Jovian magnetopause at the Earth's magnetopause by two factors 0.037 , and 0.66 . The first one is connected to the solar wind expansion with a constant speed, $V_{s w}$, which leads to a dependence proportional to $r^{-2}$ ( $r$ being the heliocentric distance). The second factor is $0.66=0.58 / 0.88$; it takes into account the difference in specific features of the gasdynamic flowing past Jupiter's and the Earth's magnetopause. If we assume at 1 AU the average value of $n_{s w E}$ to be $\sim 4 \mathrm{~cm}^{-3}$, we obtain $n_{s w J} \sim 0.14 \mathrm{~cm}^{-3}$, and for $V_{s w}=400 \mathrm{~km} / \mathrm{s}$ the value of $p_{s w J}=0.66 \cdot 0.037 p_{\text {swe }}=0.024 p_{\text {swe }}$ will be $\sim 46 \mathrm{pPa}$.

If we neglect the magnetospheric plasma contribution to the pressure balance, this dynamic pressure corresponds to the subsolar Jovian magnetopause magnetic field strength $B_{m J} \sim 9.1 \mathrm{nT}$. The Jovian dipole field alone could stop such a solar wind flow at a distance of $45.7 R_{J}\left(R_{\mathrm{J}}=7.14 \cdot 10^{7} \mathrm{~m}\right.$ is the Jovian radius), which is half the average observed subsolar magnetopause distance (for example, Joy et al. (2002) showed that for a pressure of $39 \mathrm{pPa}$, the magnetopause subsolar distance was $92 R_{J}$ ). Thus, there is more to the story at Jupiter than the simple picture of a dipole magnetic field resisting the solar wind dynamic pressure.

Just after the Pioneer 11 and Voyager flights to Jupiter (see, for example, Alexeev, 1976; Goertz, 1976a, 1979) it was revealed that the magnetic field and plasma pressure of the Jovian magnetodisc stops the solar wind flow much farther 
from the planet than Jupiter's dipole alone. It is necessary to take into account the Jovian magnetospheric plasma contribution to the pressure balance at the magnetopause. Krimigis et al. (1979) demonstrated that at the Jovian magnetopause the plasma and magnetic field contributions to the total pressure are comparable.

Several refined models simulate the magnetospheric magnetic field of Jupiter (e.g. Hill et al., 1974; Barish and Smith, 1975; Smith et al., 1975; Beard and Jackson, 1976; Goertz, 1976a, 1976b, 1979; Engle and Beard, 1980; Acuña et al., 1983; Connerney et al., 1981, 1998; Khurana, 1997). Most of them describe the data observed by some spacecraft and are applicable to limited regions of the Jovian magnetosphere. The contribution of the IMF to the solar wind-magnetosphere coupling is underestimated in these models.

In this work we will study dynamic phenomena of the Jovian magnetosphere. In the paper by Belenkaya (2004) (hereafter called Paper 1) a short review of the existing models of Jupiter's magnetospheric magnetic field is given, and a possibility of application of the constructed model to interpretation of observations (taking into account the IMF effects) was demonstrated (see also Belenkaya, 2003). In Paper 1 this model was used, but it was described only schematically. Here we give a detailed description of the model, but before we mention several recent Jovian magnetospheric models.

Khurana's (1997) model couples the internal field spherical harmonic coefficients from the Goddard Space Flight Center $O_{6}$ (Connerney, 1993) model with an Euler potential formulation of the external field. In particular, the magnetic field of the Jovian current sheet was modeled by using the Euler potential approach following Goertz et al. (1976). Khurana's (1997) model incorporated the hinging and the delay of the current sheet with radial distance, the sweep back of the magnetic field lines, and has realistic azimuthal current density profiles in the magnetosphere. Beyond a radial distance of $\sim 30 R_{J}$, the current sheet is aligned with the magnetic equator, but then departs from it toward the jovigraphic equator due to hinging. The observed and modeled delay arises due to plasma flows outward lagging behind corotation to conserve angular momentum. The radial currents bend the field lines out of meridian planes giving them a swept-back appearance, which was calculated by Khurana (1997).

The Connerney et al. (1998) model assumes axial symmetry of the magnetospheric field, including magnetodisc. Spherical harmonic models of the planetary magnetic field were obtained from in-situ magnetic field measurements. Dipole, quadrupole, octupole, and a subset of higher-degree and higher-order spherical harmonic coefficients were determined. The field due to local magnetodisc currents was modeled using an empirical model derived from Voyager observations.

Hill (2001) described a three-dimensional current system model existing in the Jovian magnetosphere, which is analogous to a Faraday disc dynamo. This current system (connected to the auroral oval) transfers planetary angular momentum to the outflowing magnetospheric plasma.
The position and the brightness of the auroral oval are determined by the mass transport rate in the Jovian magnetosphere and by the ionospheric conductance. Hill (2001) used a spin-aligned dipole magnetic field. He found the rotation frequency of magnetospheric plasma, normalized to Jupiter's rotation frequency versus dimensionless radial distance. Cowley et al. (2003a) significantly modified Hill's (2001) model using not only a dipole field, but also an empirically based current sheet model (Cowley and Bunce, 2003 ), in which the angular velocity profile of the plasma was calculated self-consistently from the Hill-Pontius theory (Pontius, 1997; Hill, 2001).

Gurnett et al. (2002) reported simultaneous observations using the Cassini and Galileo spacecraft of hectometric radio emissions and extreme ultraviolet auroral emissions from Jupiter. Their results showed that both of these emissions are triggered by interplanetary shocks propagating outward from the Sun. These shocks cause a major compression and reconfiguration of the Jovian magnetosphere, which produces strong electric fields and electron acceleration along the auroral field lines. Gurnett et al. (2002) have considered this as an incontrovertible evidence of the solar wind influence on the Jovian polar ionosphere and outer magnetosphere. It also should be noted that the influence on Jupiter's $\mathrm{H}_{3}^{+}$auroras of the solar wind was studied by for example Baron and Owen (1996) and Connerney et al. (1996).

Kivelson and Southwood (2003), using inter-spacecraft timing based on the time delay established from the interplanetary shock arrival at each spacecraft, investigated the correlation of Cassini and Galileo magnetometer measurements, offering a unique opportunity for direct study of the solar wind-Jovian interaction by using two spacecraft at once. In this work the Jovian magnetopause and bow shock positions' response to changes in the north-south component of the solar wind magnetic field was shown, a phenomenon occurring in equivalent circumstances at Earth. As Jupiter's planetary dipole moment is roughly antiparallel to that of the Earth, the effects of northward and southward interplanetary magnetic field (IMF) ought to be reversed. Thus, in the presence of northward IMF, Jupiter's dayside magnetopause should move inward. Prange et al. (2001) noted that some type of the brightest aurora onsets coincides with the arrival at the Jovian magnetopause of a coronal mass ejection (CME). Cowley et al. (2003b, using recent observations of ion flows from Doppler measurements of infrared auroras, studied plasma flows in Jupiter's high-latitude ionosphere. They found, in particular, an outermost boundary region located principally in the dawnside magnetosphere which is associated with the solar wind interaction. In the ionosphere, the region of open field lines should be a region of nearstagnation in the rest frame of the dipole, compared with surrounding regions of a few- $\mathrm{km} / \mathrm{s}$ sub-corotational flow. Walker et al. (2001) have used a three-dimensional magnetohydrodynamic simulation of the interaction between the solar wind and the Jovian magnetosphere to study the effects of the solar wind dynamic pressure and the IMF. When the pressure increases (decreases) the bow shock and magnetopause move 
toward (away from) Jupiter and the equatorial magnetic field in the middle magnetosphere becomes more dipole-like (taillike). When the IMF is southward the boundaries move away and the field becomes more dipole.

The magnetospheric model described in the present paper allows one to calculate the IMF effects (see Belenkaya, 2003 and Paper 1), and also it takes into account the magnetopause and magnetotail current fields (contrast to most of the other Jovian models). Results of the model plus Ulysses measurements during the first inbound flyby are used for the revision of the pressure balance equation at the subsolar point. Solution of this equation yields the dependence of the magnetospheric scale on the solar wind dynamic pressure.

\section{Model of the Jovian magnetospheric magnetic field}

We assume tyhat the envelope of the magnetospheric field lines fits the configuration of the magnetopause for a closed magnetosphere. The magnetopause is a current carrying surface bounded by the magnetosphere. The model presented here includes the internal magnetospheric magnetic field sources screened by the magnetopause currents, and the solar wind magnetic field penetrated into the magnetosphere.

In Paper 1 it was demonstrated that comparison with observations (e.g. Huddleston et al., 1998 and Joy et al., 2002) support our approach of approximation of the Jovian magnetopause by a paraboloid of revolution, especially up to 200 $250 R_{\mathrm{J}}$ downtail from the Jupiter. Ness et al. (1979) mentioned that the magnetopause as observed by Voyager 1 was successfully modeled by an $X$-axis symmetric parabola in Jupiter's orbital plane. From the Voyager 2 data Ness et al. (1979) obtained an analytical expression for the Jovian magnetospheric boundary: $y= \pm 10.1(68.2-x)^{1 / 2}$, where $x$ and $y$ are in units of the Jovian radius. In the model presented here (and used in Paper 1) the equation of paraboloid approximating the magnetopause is: $x / R_{\mathrm{ss}}=1-\left(y^{2}+z^{2}\right) / 2 R_{\mathrm{ss}}^{2}$. Here we use the Jovian solar-magnetospheric coordinates with the $X$-axis directed to the Sun, Jupiter's magnetic moment $\mathbf{M}_{\mathrm{J}}$ in the $X Z$ plane, and $Y$ points to dusk. $R_{\mathrm{ss}}$ is a parameter characterizing the magnetospheric scale - the jovicentric distance to the subsolar point.

Kurth et al. (2002) presented data of the radio and plasma wave science instruments on Cassini and Galileo. They made use of Cassini's flyby of Jupiter centered on 30 December 2000 , coupled with the extended Galileo orbital mission. Figure 1 shows the trajectories of Cassini (dotted curve) and Galileo (dashed and solid thick curves) near Jupiter in the December 2000-January 2001 time frame. A small part of the Cassini orbit marked by the thick solid line on the dotted curve corresponds to the time period when Cassini was inside the magnetosphere. The thick solid (dashed) curve corresponds to the time interval when Galileo was inside (outside) the magnetosphere. On 10 January 2001 almost simultaneously Cassini and Galileo observed the Jovian magnetopause. The magnetopause crossing time was determined by the disappearance of continuum radiation on

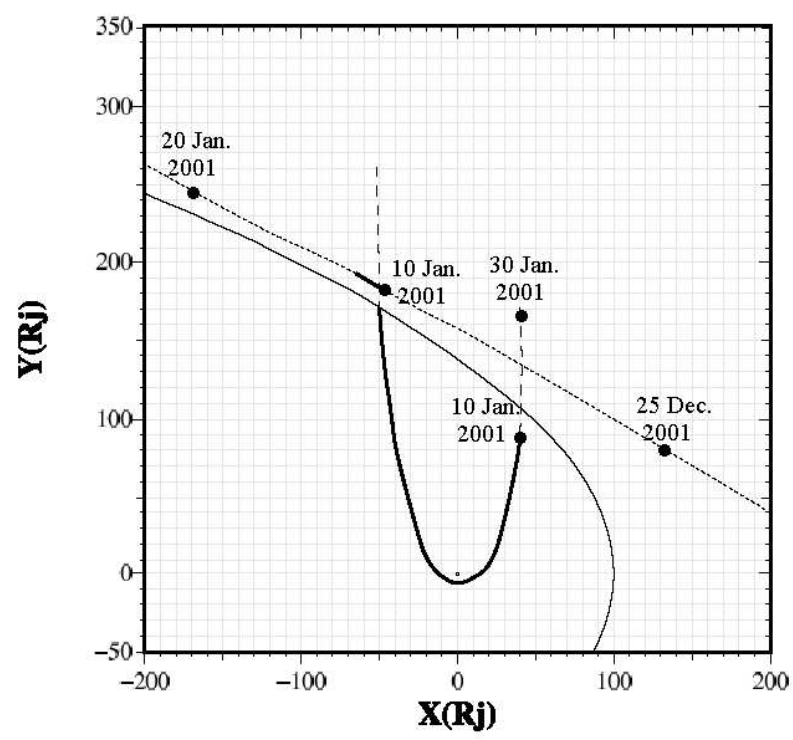

Fig. 1. The trajectories of the Cassini and Galileo during the time interval surrounding Cassini's closest approach. The coordinate system is centered on Jupiter with the positive $X$ axis directed from Jupiter to the Sun. The $Z$ axis is normal to Jupiter's orbital plane with positive north. The $Y$ axis completes an orthogonal system. The portion of the 28th and 29th Galileo orbits is shown. The portions of the orbits which correspond to the time intervals when the spacecraft were in the magnetosphere are marked by heavy curves. The magnetopause calculated in the paraboloid model is also shown. On the evening at 10 January 2001 both spacecraft crossed the magnetopause roughly simultaneously (with about 20 min delay). At this time Galileo was placed at $x=+50 R_{J}$ and Cassini was located at $x=-50 R_{J}$. The magnetopause crossing points were determinated by Kurth et al. (2002). Small, solid circles mark the beginning of the days: 25 December 2000, 10 January 2001, and 20 January 2001 on the Cassini trajectory, and 10 January 2001 (when both spacecraft crossed magnetopause), and 30 January 2001 on the Galileo orbit.

the spectrogram, changes in the magnetic field direction and spectrum, and the low energy plasma and energetic particle distributions (Kurth et al., 2002). The magnetopause crossing points shown in Fig. 1 were taken from the paper of Kurth et al. (2002), who determined an electron density of $0.05 \mathrm{~cm}^{-3}$. For the solar wind velocity of the order of $450 \mathrm{~km} / \mathrm{s}$ the derived solar wind dynamic pressure is $18 \mathrm{pPa}$. The model magnetopause (paraboloid of revolution) equatorial cross section is shown by a solid curve for the solar wind dynamic pressure $18 \mathrm{pPa}$. As it is seen from Fig. 1, the paraboloid describes rather well the shape of Jupiter's magnetopause at the distances not far from the planet.

Numerical calculations made by Engle and Beard (1980) and Engle (1991) showed the asymmetry between equatorial and noon-midnight cross sections of Jupiter's magnetosphere. The "flattened" shape of the Jovian magnetopause (compared to that of the Earth) is consistent with the disc-like magnetosphere. The number of spacecraft magnetopause crossings is too small (especially on the high-latitudes) for 
making a definite conclusion about the faithful character of the Jovian magnetopause shape. For this reason the zeroth approximation model used here does not include polar flattening or dawn-dusk asymmetry.

\subsection{Magnetospheric magnetic field sources}

To better explain our approach, we provide below a short description of the paraboloid magnetospheric field model. Utilizing the paraboloid approach introduced by Alexeev and Shabansky (1972) and Alexeev (1986), we can construct a time-dependent model of all known current systems in the magnetosphere of Jupiter. The main contributors to the model magnetospheric magnetic field are the following:

1. The intrinsic magnetic (dipole) field, as well as the shielding magnetopause currents, which confine the dipole field inside the magnetosphere of Jupiter.

2. The tail currents and their closure currents on the magnetopause.

3. The disc current and the corresponding shielding magnetopause current.

\section{The IMF penetrated into the magnetosphere.}

For completeness, we summarize Paper 1 in the description of the model. The continuity equations for the magnetic field and electric current density:

$\operatorname{div} \mathbf{B}=0 \quad \operatorname{div} \mathbf{j}=0$

are true for all model calculations. Our approach is based on the assumption that each magnetospheric current system conserves the condition $B_{n}=0$ at the magnetopause. This approach allows each current system to be changed in time with its own time scale. For a description of the dynamic phenomena in the Jovian magnetosphere our approach is preferable, as at each moment the total magnetic field component normal to the magnetopause equals zero. Inclusion of the magnetopause current shielding magnetodisc field, as well as the IMF penetrating into the magnetosphere, are new elements comparatively with the other Jovian models. Flowing pass the obstacle (magnetosphere), the magnetic field of the solar wind drapes around it and, consequently, increases in the magnetopause vicinity. Diffusion increased due to the field growth near the magnetospheric boundary leads to the penetration of the IMF through the magnetopause inside the magnetosphere. As it was shown by Alexeev (1986), the penetrated magnetic field is less than the IMF by a factor of $k_{E}$ in the case of the Earth.

We assume that currents of the magnetopause, magnetodisc, and magnetotail are concentrated in thin layers, as their thickness is much less than the characteristic scale of the Jovian magnetosphere (the subsolar distance) $\sim 100 R_{J}$. Outside these layers the magnetic field was described by corresponding scalar potentials. The magnetic field vector $\mathbf{B}_{m}$ was calculated in the Jovian solar-magnetospheric coordinate system by summing the fields of magnetospheric origin:

$$
\begin{aligned}
\mathbf{B}_{m}( & (t)=\mathbf{B}_{d}(\psi)+\mathbf{B}_{T S}\left(\psi, R_{s s}, R_{2}, B_{\mathrm{t}}\right) \\
& +\mathbf{B}_{M D}\left(\psi, B_{D C}, R_{D 1}, R_{D 2}\right)+\mathbf{B}_{s d}\left(\psi, R_{S s}\right) \\
& +\mathbf{B}_{\mathrm{SMD}}\left(\psi, R_{s s}, B_{\mathrm{DC}}, R_{D 1}, R_{D 2}\right) \\
& +\mathbf{b}\left(k_{\mathrm{J}}, \mathbf{B}_{I M F}\right) .
\end{aligned}
$$

Here $\mathbf{B}_{d}(\psi)$ is the dipole magnetic field; the field of the magnetospheric tail current system (cross-tail currents and their closure magnetopause currents) is $\mathbf{B}_{T S}\left(\psi, R_{s s}, R_{2}, B_{\mathrm{t}}\right)$; a field of the thin current disc placed near the equatorial plane is $\mathbf{B}_{M D}\left(\psi, B_{D C}, R_{D 1}, R_{D 2}\right)$; the field of currents on the magnetopause shielding the dipole field is $\mathbf{B}_{s d}\left(\psi, R_{s s}\right) ; \mathbf{B}_{\mathrm{sMD}}\left(\psi, R_{s s}, B_{\mathrm{DC}}, R_{D 1}, R_{D 2}\right)$ is the field of the currents on the magnetopause shielding the disc current field; $\mathbf{b}\left(k_{\mathrm{J}}, \mathbf{B}_{I M F}\right)$ is a part of the interplanetary magnetic field penetrating into the magnetosphere.

To calculate the Jovian magnetospheric magnetic field (Eq. 1), we have to define the time-dependent input parameters: the magnetic dipole tilt angle, $\psi$ (the angle between the $Z$ axis and the dipole axis); the distance from Jupiter's center to the subsolar point on the magnetopause, $R_{s s}$; the distances to the outer and inner edges of the magnetodisc, $R_{D 1}$ and $R_{D 2}$, respectively; the distance from the planet's center to the inner edge of the magnetospheric tail current sheet, $R_{2}$; the magnitude of the field of the tail currents at the inner edge of the tail current sheet, $B_{\mathrm{t}} / \alpha_{0}, \alpha_{0}=\sqrt{1+2 R_{2} / R_{\mathrm{Ss}}}$; the current disc magnetic field strength in the outer edge of the current disc, $B_{\mathrm{DC}}$; the interplanetary magnetic field vector, $\mathbf{B}_{I M F}$, and the coefficient of its penetration into the magnetosphere, $k_{\mathrm{J}}$. While $k_{\mathrm{J}}$ is a coefficient of IMF penetration, $1-k_{\mathrm{J}}$ is a coefficient of a partial screening of the solar wind magnetic field by the currents at the magnetopause. The coefficient of IMF penetration is often called the "efficiency of reconnection" (Cowley, 1981). The efficiency of reconnection determines the ratio of the width of a thin slab of solar wind plasma which reconnects with the magnetospheric magnetic field to the width of the total flow interacting with the magnetosphere during the passing by.

2.1.1 Approximation of the Jovian dipole field and the field of its magnetopause shielding currents

The dipole field $\mathbf{B}_{\mathrm{d}}=-\nabla U_{\mathrm{d}}$, where the scalar potential $U_{\mathrm{d}}$ is

$$
U_{\mathrm{d}}=\left(\frac{R_{\mathrm{J}}}{r}\right)^{3} \cdot B_{\mathrm{J} 0} \cdot(z \cdot \cos \psi-x \cdot \sin \psi),
$$

here $B_{\mathrm{J} 0}=4.2 \cdot 10^{5} \mathrm{nT}$ (Smith and Wenzel, 1993) is the field at the Jovian equator, and $r$ is the distance from the planet's center.

The magnetic field of the magnetopause shielding currents, $\mathbf{B}_{s d}$, was calculated similarly to that done by Alexeev and Shabansky (1972) for the terrestrial magnetosphere using the condition that the magnetic field $\mathbf{B}=\mathbf{B}_{d}+\mathbf{B}_{s d}$ is tangential to the magnetopause. The potential $U_{s d}\left(\mathbf{B}_{s d}=-\nabla U_{s d}\right)$ of the magnetopause shielding currents has been calculated as a solution of the Laplace equation with the boundary condition: 
$\mathbf{B} \cdot \mathbf{n}=0 \quad$ or $\quad \mathbf{B}_{s d} \cdot \mathbf{n}=-\mathbf{B}_{d} \cdot \mathbf{n}$,

where $\mathbf{n}$ is a normal to the magnetopause.

Due to the paraboloid axial symmetry, the potential $U_{s d}$ has a simple representation in the spherical jovicentric coordinate system $r, \theta^{\prime}, \varphi^{\prime}$. The polar axis of this coordinate system is the Jupiter-Sun line, $\theta^{\prime}$ being the polar angle $\left(\cos \theta^{\prime}=x / r\right)$, and the azimuthal angle $\varphi^{\prime}$ is counted from the $\mathrm{XZ}$ plane of the Jovian solar-magnetospheric coordinate system to the dusk part $(y>0)$ of the magnetospheric equatorial plane. In these coordinates, the scalar potential $U_{\mathrm{sd}}$ is expanded in a series of spherical harmonics:

$$
\begin{aligned}
U_{\mathrm{sd}} & =-B_{\mathrm{J} 0} \frac{R_{\mathrm{J}}^{3}}{R_{\mathrm{ss}}^{2}} \sum_{n=1}^{\infty}\left[d_{n}^{\|} \sin \psi P_{n}\left(\cos \theta^{\prime}\right)\right. \\
& \left.+d_{n}^{\perp} \cos \psi \cos \varphi^{\prime} P_{n}^{1}\left(\cos \theta^{\prime}\right)\right]\left(\frac{r}{R_{\mathrm{ss}}}\right)^{n},
\end{aligned}
$$

where

$$
\begin{aligned}
& P_{n}(x)=\frac{1}{2^{n} n !} \cdot \frac{d^{n}\left(x^{2}-1\right)^{n}}{d x^{n}}, \quad \text { and } \\
& P_{n}^{1}(x)=\sqrt{1-x^{2}} \cdot \frac{d P_{n}}{d x}
\end{aligned}
$$

are the Legendre polynomials and the associated Legendre functions, respectively; $d_{n}^{\|}$and $d_{n}^{\perp}$ are the dimensionless coefficients; these coefficients describe the magnetic field of the currents induced on the magnetopause by the dipole perpendicular and parallel to the solar wind flow, respectively. The first six dimensionless coefficients $d_{n}^{\|}$and $d_{n}^{\perp}$ are listed in the second and third columns of Table 1; these coefficients describe the magnetic field of the currents induced on the magnetopause by the dipole perpendicular and parallel to the solar wind flow, respectively.

The expansion parameter of Eq. (2) is $r / R_{s s}$, therefore, this equation can be used only up to $r \leq R_{\mathrm{ss}}$. Over the distant nightside it is more convenient to present the sum of potentials $U_{\mathrm{d}}+U_{\mathrm{sd}}$ in the parabolic coordinates as expansion by the Bessel functions (or parabolic harmonics):

$$
\begin{aligned}
U_{\mathrm{d}} & +U_{\mathrm{sd}}=\sin \psi \sum_{n=1}^{\infty} D_{n} J_{\circ}\left(\lambda_{0 n} \alpha\right) K_{\circ}\left(\lambda_{0 n} \alpha\right) \\
& +\cos \psi \cos \phi \sum_{n=1}^{\infty} G_{n} J_{1}\left(\lambda_{1 n} \beta\right) K_{1}\left(\lambda_{1 n} \alpha\right) .
\end{aligned}
$$

In the parabolic coordinates, the Laplacian has the eigenfunctions of the form:

$J_{m}\left(\lambda_{m n} \beta\right) K_{m}\left(\lambda_{m n} \alpha\right) \cos m \varphi^{\prime}$,

where $J_{m}$ and $K_{m}$ are the Bessel functions of the first kind of the real and imaginary arguments, respectively. The orthogonal parabolic coordinates $\alpha, \beta, \varphi^{\prime}$ are defined in the $(x, y, z)$ solar-magnetospheric Cartesian coordinates as follows:

$$
\begin{aligned}
& \beta^{2}-\alpha^{2}+1=2 \frac{x}{R_{\mathrm{ss}}}, \\
& \alpha \beta \sin \varphi^{\prime}=\frac{y}{R_{\mathrm{ss}}}, \\
& \alpha \beta \cos \varphi^{\prime}=\frac{z}{R_{\mathrm{ss}}} .
\end{aligned}
$$

Table 1. The coefficients of expansion of the potential $U_{c f}$ in spherical harmonics $\left(d_{n}^{\perp}, d_{n}^{\|}\right)$and in Bessel functions $\left(D_{n}, G_{n}\right)$.

\begin{tabular}{rrrll}
\hline$n$ & $d_{n}^{\perp}$ & $d_{n}^{\|}$ & $D_{n}$ & $G_{n}$ \\
\hline 1 & 0.6497 & 0.9403 & $9.46305 \cdot 10^{3}$ & $-1.31869 \cdot 10^{3}$ \\
2 & 0.2165 & -0.4650 & $1.07980 \cdot 10^{7}$ & $-1.90098 \cdot 10^{5}$ \\
3 & 0.0434 & 0.1293 & $6.52950 \cdot 10^{8}$ & $-9.60338 \cdot 10^{6}$ \\
4 & -0.0008 & -0.0148 & $3.01584 \cdot 10^{10}$ & $-3.69794 \cdot 10^{8}$ \\
5 & -0.0049 & -0.0160 & $1.19816 \cdot 10^{12}$ & $-1.25022 \cdot 10^{10}$ \\
6 & -0.0022 & -0.0225 & - & - \\
\hline
\end{tabular}

In Eq. (4) $\lambda_{0 n}, \lambda_{1 n}$ are solutions of the equations $J_{0}^{\prime}(x)=0$ and $J_{1}^{\prime}(x)=0$, respectively. We will use Eq. (2) for the case $\alpha<\alpha_{0}$, and Eq. (4) for the case $\alpha>\alpha_{0}$. The value of $\alpha_{0}$ is determined by the distance to the inner edge of the jovimagnetic tail current sheet, $R_{2}$ (see Sect. 2.1.2):

$\alpha_{0}=\sqrt{1+\frac{2 R_{2}}{R_{\mathrm{ss}}}}$.

In the parabolic coordinates the magnetospheric boundary is taken to be the surface $\beta=1$. The subsolar point at the magnetopause is at $\left(R_{\mathrm{Ss}}, 0,0\right)$ or at $\alpha=0, \beta=1$. The surfaces $\alpha=$ constant are the confocal paraboloids of revolution which are open toward the dayside, and the constant $\beta$ surfaces are the paraboloids with the same focus but open toward the nightside. Jupiter is at the origin of the $(x, y, z)$ coordinate system and at $\alpha=1, \beta=0$. In numerical calculations, we used the first six coefficients $D_{n}$ and $G_{n}$ (see Eq. 4), presented in the fourth and fifth columns of Table 1 .

Figure 2 shows the Jovian dipole magnetic field and the field of the magnetopause currents screening it in the noonmidnight cross section of the Jovian magnetosphere. The dipole and its screening currents create in the subsolar point a magnetic field equal to $1.31 \mathrm{nT}$, which is significantly less than the total magnetospheric magnetic field measured at the Jovian subsolar point $(\geq 4 \mathrm{nT})$.

A solution of the problem of the dipole screening by the paraboloid of revolution was obtained by Alexeev and Shabansky (1972). Greene and Miller (1994) solved the same problem for the arbitrary magnetopause tail flaring angle. Both solutions have been presented by integral transformations. To shorten the magnetic field calculation time, the scalar potential of the magnetic field was expanded into a series of orthogonal functions (spherical functions inside the sphere of radius $R_{s s}$, and Bessel functions in the magnetospheric tail). The coefficients used in the calculations are given in Table 1. An initial integral representation satisfies the boundary condition $B_{n}=0$ at the magnetopause with accuracy $\left|B_{n} / B\right| \leq 10^{-4}-10^{-6}$ determined by the numerical integration accuracy and the accuracy of the Bessel function calculations. The chosen number of the series terms provides the same accuracy. The maximum deviation $\Delta B$ between the result of integral representation and the series calculation 


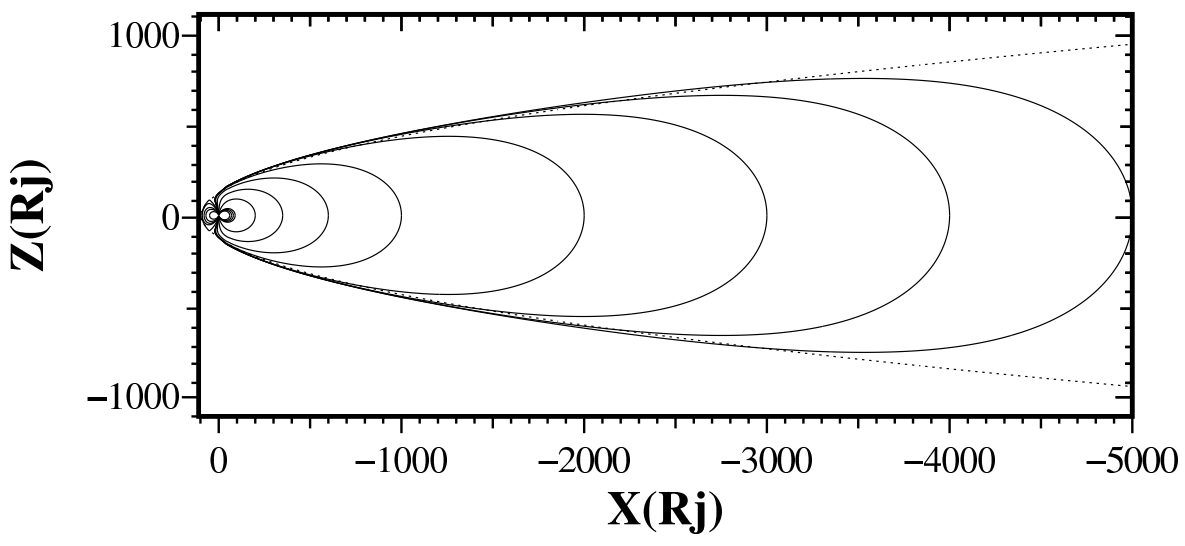

Fig. 2. The Jovian dipole field and the field of the Chapman-Ferraro magnetopause current which shields the dipole field in the noon-midnight section of Jupiter's magnetosphere. The normal to the magnetopause component of the total field $\mathbf{B}_{n}=0$. Outside the magnetopause, the magnetic field equals zero. The magnetopause is marked by the dotted curve. Along $X$ and $Y$ axes there are distances in Jovian radii. Model parameters are: $\Psi=0 ; R_{\mathrm{Ss}}=100 R_{\mathrm{J}}$. At the distant low-latitude, the nightside magnetosphere magnetic field is southward.

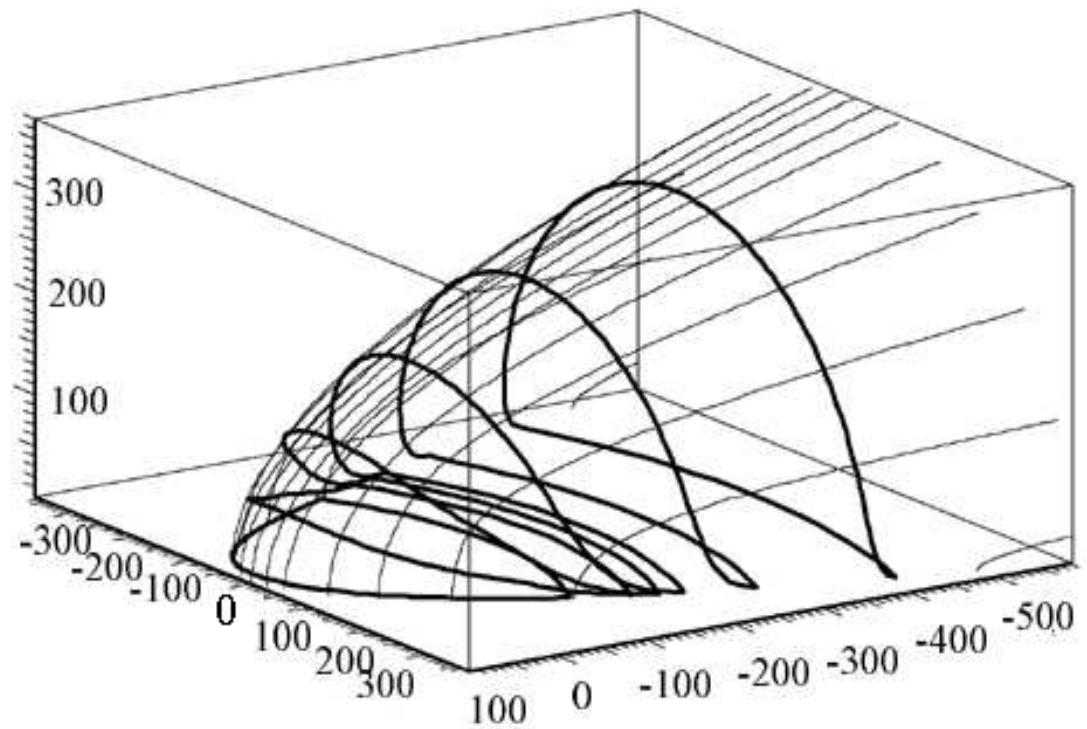

Fig. 3. The tail current system is shown. In the equatorial tail, the current flows from dusk to dawn. This current is closed by the northern and southern magnetopause currents. The current lines are shown by heavy curves. Thin curves mark the magnetic field lines lying on the magnetopause. Parameters of the tail current system used in the calculations are $R_{S s}=100 R_{J}$ and $R_{2}=65 R_{J}$. Under condition of the absence of the component of the tail current system magnetic field normal to the magnetopause, the closure currents from the inner part of the tail current should go to the dayside magnetopause. This is a consequence of very high conductivity of the solar wind plasma which preserves penetration of the magnetic field outside the magnetosphere.

can be estimated as $\Delta B / B \leq 10^{-4}$. As we use the orthogonal function series, it is possible to improve the accuracy by adding additional highest terms of expansion. The coefficients shown in Table 1 are not changed by this procedure.

\subsubsection{Magnetic field of the tail current system}

We used a model of the tail current system magnetic field which takes into account a finite thickness of the current sheet, $2 d$. The current sheet is placed at $\alpha>\alpha_{0}$ and $0<\beta<\beta_{c}\left(\varphi^{\prime}\right)$, where the function $\beta_{c}\left(\varphi^{\prime}\right)$ is determined by

$$
\begin{aligned}
& d_{0}=\frac{d}{\alpha_{0} R_{\mathrm{Ss}}} \text { and } \varphi^{\prime}: \\
& \beta_{c}\left(\varphi^{\prime}\right)=\left\{\begin{array}{cc}
\frac{d_{0}}{\left|\cos \varphi^{\prime}\right|} & \text { for }\left|\cos \varphi^{\prime}\right| \geq d_{0}, \\
1 & \text { for } d_{0} \geq\left|\cos \varphi^{\prime}\right| .
\end{array}\right.
\end{aligned}
$$

The shift, $z_{0}$, of the current sheet with respect to the solarmagnetospheric XY plane is (see also Alexeev and Shabansky, 1972):

$$
z_{0}=R_{\mathrm{SS}} \sin 2 \psi\left(3+\sin ^{2} \psi\right)^{-1},
$$


$z_{0}=0$ when $\psi=0$; this is a case which we will consider hereafter. For simplicity we will treat the case of a spin-aligned dipole magnetic field (aligned with axis $\mathrm{Z}$ of the jovicentric solar-magnetospheric coordinate system X, Y, Z).

The magnetotail current system includes the dawnward currents in the neutral sheet, which are tangential to the paraboloids $\alpha=$ const, and the closure currents on the magnetopause, which screen the outer space from the magnetic field of the tail current system (see Fig. 3).

Inside the current sheet, the magnetic field of the tail current system is a sum of two terms:

$\mathbf{B}_{\mathrm{TS}}=\mathbf{B}_{1}+\mathbf{B}_{2}$,

where $\mathbf{B}_{1}=-B_{\mathrm{t}} R_{\mathrm{ss}} \nabla \mathbf{U}_{\mathrm{t} 1}$, and $2 B_{\mathrm{t}} / \alpha_{0}$ is the field drop across the neutral sheet at its inner edge. The scalar potential $U_{\mathrm{t} 1}$ defines a component of the tail magnetic field perpendicular to the equatorial plane

$U_{\mathrm{t} 1}\left(\alpha, \beta, \varphi^{\prime}\right)=\sum_{k, n=1}^{\infty} c_{n k} \cos n \varphi^{\prime} J_{n}\left(\lambda_{n k} \beta\right) K_{n}\left(\lambda_{n k} \alpha\right)$,

where the $k$-th solution of the equation $\frac{d J_{n}(x)}{d x}=0$ is $\lambda_{n k}$.

The current density vector is proportional to $\nabla \times \mathbf{B}_{2}$. It is tangential to the paraboloid $\alpha=$ const and parallel to the equatorial plane. Magnetic field $\mathbf{B}_{2}$ is a solution for equation:

$\nabla \times \mathbf{B}_{2}=\mu_{0} \mathbf{j}_{\mathbf{t}}$

where $\mathbf{j}_{\mathrm{t}}$ is the current density in the tail current sheet and $\mathbf{B}_{2}$ is a solenoidal part of the magnetic field. $\mathbf{B}_{2}$ is found as a partial solution of the vector potential problem:

$B_{2 \alpha}=B_{\mathrm{t}} \frac{\alpha_{0}}{\alpha} \frac{\beta}{\beta_{\mathrm{c}}(0)} \frac{\cos \varphi^{\prime}}{\sqrt{\alpha^{2}+\beta^{2}}} ; \quad B_{2 \beta}=0 ; \quad B_{2 \varphi^{\prime}}=0$.

Outside the current sheet, the dimensionless scalar potential $u_{t}=\frac{U_{\mathrm{t}}}{B_{\mathrm{t}} R_{\mathrm{Ss}}}$ of the magnetic field of the tail current system is:

$u_{t}=\left\{\begin{array}{c}\sum_{k, n=1}^{\infty} b_{n k} \cos n \varphi^{\prime} J_{n}\left(\lambda_{n k} \beta\right) I_{n}\left(\lambda_{n k} \alpha\right) \\ \text { for } \alpha<\alpha_{0}, 1 \geq \beta>0, \\ \alpha_{0} \ln \alpha \operatorname{sign}\left(\frac{\pi}{2}-\left|\varphi^{\prime}\right|\right) \\ +U_{\mathrm{t} 1}\left(\alpha, \beta, \varphi^{\prime}\right) \\ \quad \text { for } \alpha \geq \alpha_{0}, \quad 1 \geq \beta \geq \beta_{\mathrm{c}}\left(\varphi^{\prime}\right) .\end{array}\right.$
Table 2. Numerical values of the coefficients $f_{n k}$ of scalar potential $U_{t}$ of the tail current system.

\begin{tabular}{clcll}
\hline $\mathrm{k} \backslash \mathrm{n}$ & \multicolumn{1}{c}{1} & \multicolumn{1}{c}{3} & \multicolumn{1}{c}{5} & \multicolumn{1}{c}{7} \\
\hline 1 & 2.0635 & -0.4437 & 0.2949 & -0.280 \\
2 & 0.108665 & -0.053383 & 0.041799 & -0.04171 \\
3 & 0.029803 & -0.017021 & 0.012939 & -0.01203 \\
4 & 0.012946 & -0.008451 & 0.006415 & -0.00537 \\
5 & 0.006536 & -0.004620 & 0.003708 & -0.00309 \\
\hline
\end{tabular}

In Equations (9) and (11) the coefficients $b_{n k}$ and $c_{n k}$ are defined by $f_{n k}$ as:

$$
\begin{aligned}
& b_{n k}=2 \lambda_{n k} f_{n k}\left[1+\lambda_{n k}^{2} I_{n}\left(\lambda_{n k} \alpha_{0}\right) K_{n}^{\prime}\left(\lambda_{n k} \alpha_{0}\right)\right], \\
& c_{n k}=2 f_{n k} \lambda_{n k}^{3} I_{n}\left(\lambda_{n k} \alpha_{0}\right) I_{n}^{\prime}\left(\lambda_{n k} \alpha_{0}\right),
\end{aligned}
$$

and

$$
\begin{gathered}
f_{n k}=2\left\{\frac{\int_{-\frac{\pi}{2}}^{\frac{\pi}{2}} \cos n \phi \int_{\beta_{c}(\varphi)}^{1} J_{n}\left(\lambda_{n k} \beta\right) \beta d \beta d \varphi}{\pi\left(\lambda_{n k}^{2}-n^{2}\right) J_{n}^{2}\left(\lambda_{n k}\right) I_{n}^{\prime}\left(\lambda_{n k} \alpha_{0}\right)}\right. \\
\left.+\frac{\int_{-\frac{\pi}{2}}^{\frac{\pi}{2}} \cos n \phi \cos \phi \int_{0}^{\beta_{c}(\phi)} J_{n}\left(\lambda_{n k} \beta\right) \beta d \beta d \varphi}{\beta_{c}(0) \pi\left(\lambda_{n k}^{2}-n^{2}\right) J_{n}^{2}\left(\lambda_{n k}\right) I_{n}^{\prime}\left(\lambda_{n k} \alpha_{0}\right)}\right\} .
\end{gathered}
$$

Numerical values of $f_{n k}$ are presented in Table 2 for $\alpha_{0}=\sqrt{2.4}$ (in this case $R_{2}=0.7 R_{\mathrm{ss}}$ ) and $n=2 m+1$. For $n=2 m$ the coefficients $f_{n k}$ are equal to zero.

A good approximation for the tail current system magnetic field along the $X$ axis is given by Alexeev et al. (2000):

$B_{\mathrm{TS}}=\frac{B_{\mathrm{t}}}{\alpha_{0}}\left\{\begin{array}{l}\exp \left\{-\frac{x+R_{2}}{R_{\mathrm{SS}}}\right\} \text { for } x>-R_{2}, \\ \exp \left\{2 \frac{x+R_{2}}{R_{\mathrm{SS}}}\right\} \text { for } x<-R_{2} .\end{array}\right.$

Figure 4 shows the magnetic field of the tail current system in the noon-midnight meridional cross section of the Jovian magnetosphere. The tail current system was calculated under the condition that its magnetic field component normal to the magnetopause equals zero. This condition determines a unique solution, in which closure currents from the inner part of the tail current sheet are closed at the subsolar magnetopause (see Fig. 3). The direction of these currents at the noon magnetopause is opposite to the direction of the magnetopause currents that shield the dipole field. The calculated magnetic field strength at the subsolar point of the magnetopause is $B_{\mathrm{ss}}=0.05 \mathrm{nT}$ (the contribution of the tail current system to the magnetic field at the subsolar magnetopause is very small). 


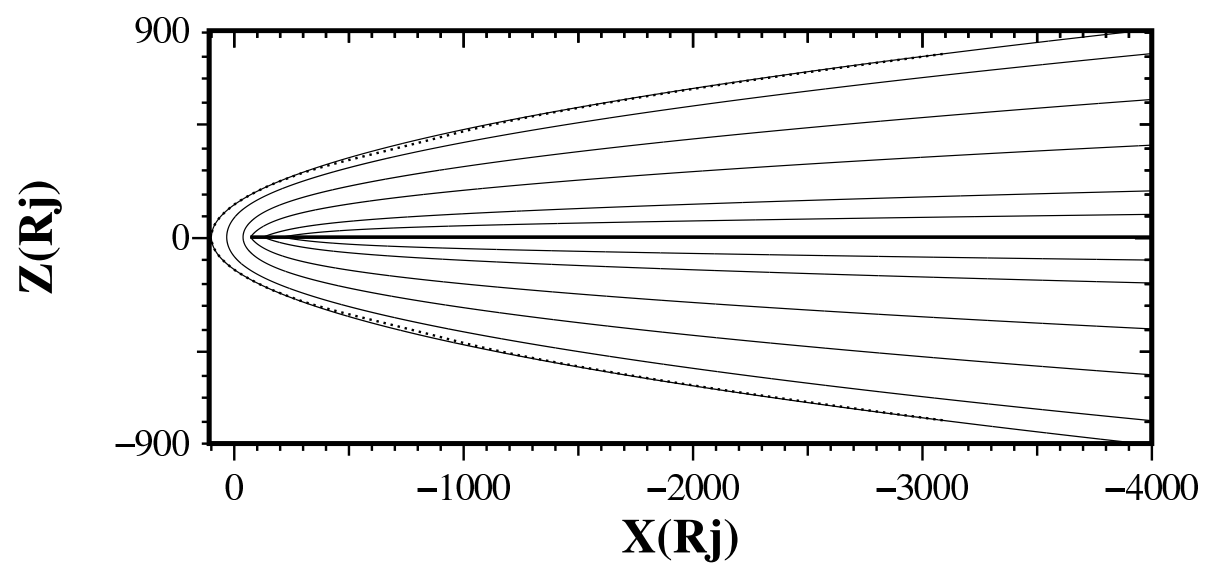

Fig. 4. Noon-midnight meridional cross section. The magnetic field lines of the Jovian tail current system are shown. The magnetic field points north at the dayside equator. The chosen values of the model parameters $R_{\mathrm{ss}}, R_{2}$, and $B_{\mathrm{t}}$ are $100 R_{\mathrm{J}}, 65 R_{\mathrm{J}}$, and $-0.5 \mathrm{nT}$, respectively.

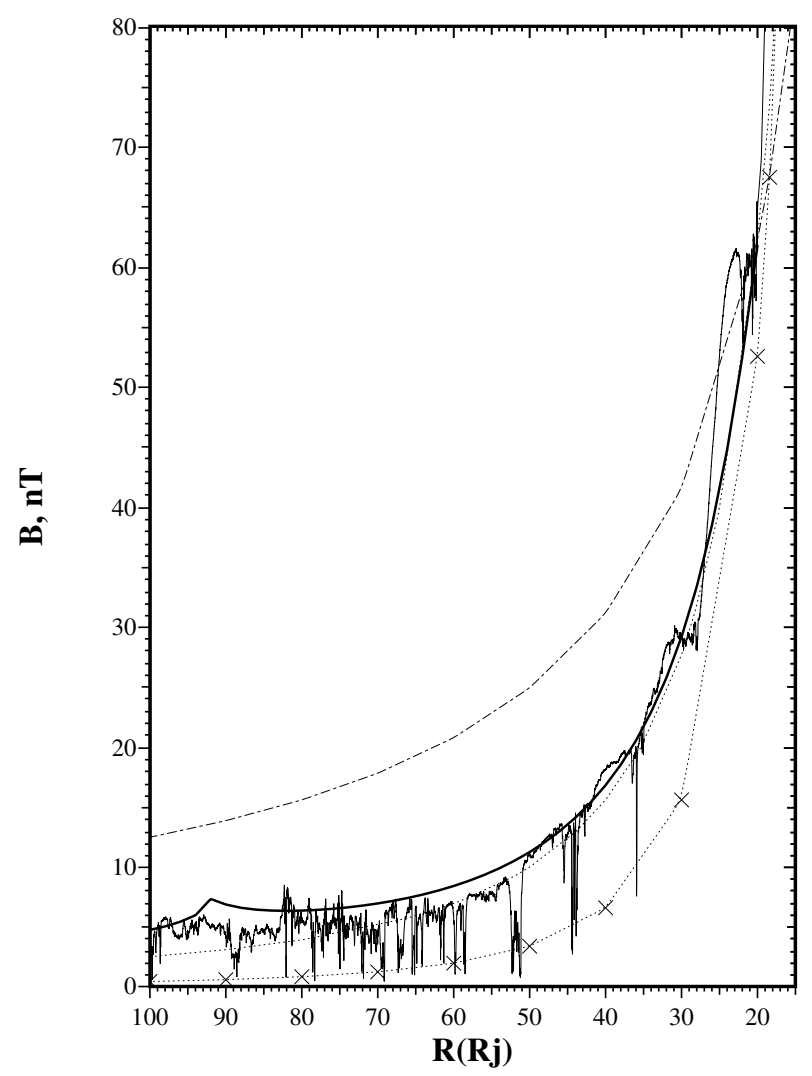

Fig. 5. Measured by Ulysses the Jovian magnetospheric magnetic field dependent on the radial distance $r$ (Cowley et al., 1996) is marked by a solid curve. For comparison the magnetic field strength calculated by the present model (heavy curve), the $r^{-2}$ power-law (dotted curve), the $r^{-1}$ power-law (dotted-dashed curve), the $r^{-3}$ Jovian dipole power-law (dotted curve marked by crosses) are also shown. The power law curves were normalized on the measured field strength at $20 R_{J}: 62.2 \mathrm{nT}$. Data (PI A. Balogh, Imperial College, London, UK) have been received by using COHOWEB system, NSSDC, NASA/GSFC, USA.

\subsubsection{Magnetic field of the current disc}

At the present time a lot of magnetodisc magnetic field models exist (Smith et al., 1974; Goertz et al., 1976; Goertz, 1979; Barbosa et al., 1979; Engle and Beard, 1980; Behannon et al., 1981; Connerney et al., 1981, 1998; Bespalov and Davidenko, 1994; Khurana, 1997). However, we use a simple model which is easilyeasily included in the paraboloid model of Jupiter's magnetosphere and reflects the salient features of the magnetodisc structure: the magnitude of the field decreases with the distance from Jupiter more slowly than for a dipole field and the direction of the field near the equatorial plane in the middle magnetosphere is radial.

We use a spherical coordinate system with the axis $Z$ parallel to the dipole axis, the polar angle, $\theta$, and the azimuthal angle, $\varphi$, counted in the planet rotation direction. The rigid plasma disc is placed in the magnetic equatorial plane. The distances to the inner and outer edges of the magnetodisc are $R_{\mathrm{D} 2}$ and $R_{\mathrm{D} 1}$, respectively. The azimuthal symmetry is suggested about the magnetic dipole axis. In our model an effective radial outflow of magnetospheric plasma is taken into account by including the magnetodisc field.

The azimuthal magnetodisc current, $\mathbf{j}_{\mathrm{MD}} \varphi$, exists only inside the disc and is directed to dusk in the dayside, and to dawn in the nightside. Caudal (1986) showed that a selfconsistent model of Jupiter's disc including the effects of centrifugal force and pressure gives $1 / r$ magnetodisc current dependence. However, here following Barish and Smith (1975) and Beard and Jackson (1976), we assume a $1 / r^{2}$ current disc dependence. In this case, the magnetic flux of the disc field, $F l_{\mathrm{MD}}=B_{\mathrm{MD}} r \cdot 2 \pi r^{2}$, across the Southern or Northern Hemisphere is constant. As it is seen from Fig. 5, this dependence fits well to the Ulysses data.

For the Jovian magnetospheric magnetic field B Behannon et al. (1981) determined the simple power law parameterization $B \approx 90 \mathrm{nT}\left(\frac{20}{r\left(R_{J}\right)}\right)^{1.7}$ for the outbound Pioneer 10 pass (it should be noted that this flight took place at different 
local times in comparison with the inbound flights). In Fig. 5 we compare several power law parameterizations with the Ulysses inbound pass data (Cowley et al., 1996). All model curves are normalized to the Ulysses field strength $(\sim 62.2 \mathrm{nT})$ at $r=20 R_{J}$. It is seen that our approach (current disc dependence proportional to $r^{-2}$ ) gives the model magnetic field (heavy solid curve in Fig. 5), which coincides very well with the Ulysses data at magnetodisc distances $\left(\sim 20-90 R_{J}\right)$. The planetary dipole field (dotted with crosses curve) gives only a small part of the total field in the outer magnetosphere. Some underestimation near the magnetopause of the total magnetospheric field by the $r^{-2}$ law shows that in this region the magnetopause current field (about $2.8 \mathrm{nT}$ ) is essential.

A vector potential $\mathbf{A}_{\mathrm{MD}}$ of the magnetodisc magnetic field $\mathbf{B}_{\mathrm{MD}}$ is introduced:

$\mathbf{B}_{\mathrm{MD}}=\nabla \times \mathbf{A}_{\mathrm{MD}}$.

If we assume that in magnetodisc only the azimuthal current, $\mathbf{j}_{\mathrm{MD}} \varphi$, exists, the vector-potential has only one non-zero component, $A_{\mathrm{MD}} \varphi$. In a current-free region, $A_{\mathrm{MD}} \varphi$ is a solution of equation: $\nabla \times \nabla \times A_{\mathrm{MD}} \varphi=0$, which in spherical coordinates looks like:

$r \frac{\partial^{2}\left(r A_{\mathrm{MD} \varphi}\right)}{\partial r^{2}}+\frac{\partial}{\partial \theta}\left(\frac{1}{\sin \theta} \frac{\partial\left(A_{\mathrm{MD} \varphi} \sin \theta\right)}{\partial \theta}\right)=0$.

Assuming a separation of variables, we can find solutions in the form

$r^{n} \cdot P_{n}^{1}(\cos \theta)$ and $\frac{P_{n}^{1}(\cos \theta)}{r^{n+1}}$,

$n=1,2, \ldots, \infty$,

where $P_{n}^{1}(\cos \theta)$ are the associated Legendre polynomial functions. These solutions provide a continuity of the magnetic field at the edges of the magnetodisc. Discontinuity of the magnetic field caused by the disc current is described by another solution of Eq. (14):

$A_{\mathrm{MD} \varphi}^{(1)}=B_{\mathrm{DC}} \frac{R_{\mathrm{D} 1}^{2}}{r}\left\{\begin{array}{l}\tan \frac{\theta}{2} \text { for } \quad 0 \leq \cos \theta, \\ \cot \frac{\theta}{2} \text { for } \quad \cos \theta \leq 0 .\end{array}\right.$

This solution yields a drop of $B_{\mathrm{MD}} r$ at the equatorial plane $(\theta=\pi / 2):\left.\quad\left\{B_{\mathrm{MD} r}\right\}\right|_{\theta / 2}$. For $j_{\mathrm{MD} \varphi} \sim 1 / r^{2}$, inside the disc $A_{\mathrm{MD} \varphi} \sim 1 / r, B_{\mathrm{MD} \theta}=0$, and $B_{\mathrm{MD} r} \sim r^{-2}$.

To construct a solution for the magnetic field of the disc current we use a principle of superposition of

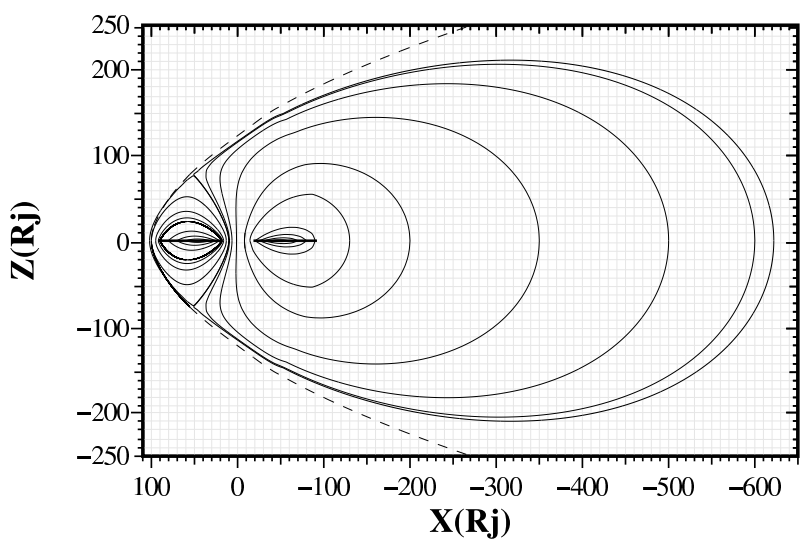

Fig. 6. Noon-midnight meridional cross section. The magnetic fields are caused by the Jovian magnetodisc and its screening magnetopause current. The magnetic field points south at the equator. The magnetopause is shown by the dashed line. The chosen parameters: $R_{\mathrm{SS}}=100 R_{\mathrm{J}} ; R_{\mathrm{D} 1}=92.07 R_{\mathrm{J}} ; R_{\mathrm{D} 2}=18.4 R_{\mathrm{J}} ; B_{\mathrm{DC}}=2.5 \mathrm{nT}$.

solutions Eqs. (15) and (16). The vector potential $A_{\mathrm{MD}} \varphi$ can be written as:

$A_{\mathrm{MD} \varphi}=\left\{\begin{array}{c}\sum_{k=0}^{\infty} F_{1 k}\left(\frac{R_{\mathrm{D} 1}}{r}\right)^{2 k+2} P_{2 k+1}^{1}(\cos \theta) \\ \text { for } R_{\mathrm{D} 1} \leq r, \\ A_{\mathrm{MD} \varphi}^{(1)}+\sum_{k=0}^{\infty} P_{2 k+1}^{1}(\cos \theta)\left[F_{2 k}\left(\frac{r}{R_{\mathrm{D} 1}}\right)^{2 k+1}\right. \\ \left.+G_{1 k}\left(\frac{R_{\mathrm{D} 2}}{r}\right)^{2 k+2}\right] \text { for } R_{\mathrm{D} 2}<r<R_{\mathrm{D} 1}, \\ \sum_{k=0}^{\infty} G_{2 k}\left(\frac{r}{R_{\mathrm{D} 2}}\right)^{2 k+1} P_{2 k+1}^{1}(\cos \theta) \\ \text { for } r \leq R_{\mathrm{D} 2} .\end{array}\right.$

To calculate the coefficients $F_{1 k}, F_{2 k}$ and $G_{1 k}, G_{2 k}$, we use the continuity conditions for $B_{\mathrm{MD} \theta}$ and $B_{\mathrm{MD}} r\left(B_{\mathrm{MD} \varphi} \equiv 0\right.$ in the considered model) at the edges of the disc (at $r=R_{\mathrm{D} 1}$ and $\left.r=R_{\mathrm{D} 2}\right)$.

The resulting expression for $B_{\mathrm{MD} r}=\frac{\partial\left(A_{\mathrm{MD}} \varphi \sin \theta\right)}{r \sin \theta \partial \theta}$ is

$B_{\mathrm{MD} r}=$
$B_{\mathrm{DC}}\left\{\begin{array}{l}\sum_{k=0}^{\infty} a_{2 k}\left(1-\rho_{0}^{2 k+1}\right) \frac{P_{2 k+1}(\cos \theta)}{\rho^{2 k+3}} \\ \text { for } R_{\mathrm{D} 1 \leq r}, \\ \frac{\operatorname{sign}(\cos \theta)}{\rho^{2}}+\sum_{k=0}^{\infty} P_{2 k+1}(\cos \theta)\left(a_{2 k+2}\right. \\ \left.-a_{2 k} \frac{\rho_{0}^{2 k+1}}{\rho^{4 k+3}}\right) \rho^{2 k} \text { for } R_{\mathrm{D} 2} \leq r \leq R_{\mathrm{D} 1}, \\ \sum_{k=0}^{\infty} a_{2 k+2}\left(1-\frac{1}{\rho_{0}^{2 k+2}}\right) \rho^{2 k} P_{2 k+1}(\cos \theta) \\ \text { for } r \leq R_{\mathrm{D} 2} .\end{array}\right.$ 
Here we introduce coefficients $a_{2 k}=\left.P_{2 k}(\cos \theta)\right|_{\theta=\pi / 2}=$ $\frac{(-1)^{k}}{2^{k} k !} 1 \cdot 3 \cdots(2 k-1)$, a dimensionless distance $\rho=\frac{r}{R_{\mathrm{D} 1}}$, and $\rho_{0}=\frac{R_{\mathrm{D} 2}}{R_{\mathrm{D} 1}}$. The function sign $(\cos \theta)$ could be expanded in a series of Legendre polynomials:

$\operatorname{sign}(\cos \theta)=\sum_{k=0}^{\infty} \frac{a_{2 k}}{2 k+2} P_{2 k+1}(\cos \theta)$,

and calculations were performed for $k \leq 50$.

The other component of the magnetodisc magnetic field $B_{\mathrm{MD} \theta}=-\frac{\partial\left(r A_{\mathrm{MD} \varphi}\right)}{r \partial r}$ can be expressed as

$B_{\mathrm{MD} \theta}=$

$B_{\mathrm{DC}}\left\{\begin{array}{c}\sum_{k=0}^{\infty} \frac{a_{2 k}}{2 k+2}\left(1-\rho_{0}^{2 k+1}\right) \frac{P_{2 k+1}^{1}(\cos \theta)}{\rho^{2 k+3}} \\ \text { for } R_{\mathrm{D} 1 \leq r,} \leq \frac{a_{2 k}}{2 k+2}\left(\rho^{2 k}-\frac{\rho_{0}^{2 k+1}}{\rho^{2 k+3}}\right) P_{2 k+1}^{1}(\cos \theta) \\ \text { for } R_{\mathrm{D} 2} \leq r \leq R_{\mathrm{D} 1}, \\ \sum_{k=0}^{\infty} \frac{a_{2 k}}{2 k+2}\left(\begin{array}{c}\left.\rho_{0}^{2 k}-\frac{1}{\rho_{0}^{2}}\right) \frac{\rho^{2 k}}{\rho_{0}^{2 k}} P_{2 k+1}^{1}(\cos \theta) \\ \text { for } r \leq R_{\mathrm{D} 2} .\end{array}\right.\end{array}\right.$

For $R_{\mathrm{D} 1}<r$ the first term in the sum for $B_{\mathrm{MD} \theta}$ (Eq. 20) corresponding to $k=0$ and $\theta=\pi / 2$ is equal to

$\left.B_{\mathrm{MD} \theta}\right|_{k=0, \theta=\pi / 2}=\frac{B_{\mathrm{DC}}}{2 \rho^{3}}\left(1-\rho_{0}\right)=\frac{M_{\mathrm{MD}}}{r^{3}}$,

where

$M_{\mathrm{MD}}=\frac{B_{\mathrm{DC}}}{2} R_{\mathrm{D} 1}^{3}\left(1-\rho_{0}\right)$

is an effective magnetic moment of the magnetodisc field for $R_{\mathrm{D} 1}<r$.

The problem of determination of the magnetopause current screening of the magnetodisc magnetic field is solved similarly to the problem for Jupiter's dipole field. Outside the outer edge of the magnetodisc its magnetic field is similar to the dipole field with an effective magnetic moment $M_{\mathrm{MD}}$ Eq. (22). So, in zeroth approximation, the field of the magnetodisc screening current is equal to the Jovian dipole screening current field multiplied by a factor $M_{\mathrm{MD}} / M_{\mathrm{J}}\left(M_{\mathrm{J}}=4.2 \cdot 10^{5} \mathrm{nT} \cdot R_{\mathrm{J}}^{3}\right.$, e.g. Smith and Wenzel, 1993).

The model magnetic field of the magnetodisc and its screening currents is demonstrated in Fig. 6 in the noonmidnight cross section. From the model calculations it follows that for the chosen parameters of the model, at the subsolar point the ratio of the magnetic field strengths of the magnetodisc and its screening current to Jupiter's dipole is 2.62. It means that the effective magnetic moment of the magnetodisc field exceeds Jupiter's dipole moment by this factor. The calculated magnetic field strength in the subsolar magnetopause is $B_{\mathrm{ss}}=3.43 \mathrm{nT}$.

\section{Closed Jovian magnetosphere}

Figure 7 presents the calculated net magnetic field in the closed Jovian magnetosphere including all described current systems. In this paper our primnary focus is on the pressure balance at the subsolar point (see below Sect. 3.1). That's why we justify the model parameters $R_{s S}=100 R_{J}$; $R_{2}=65 R_{J} ; B_{t}=-2.5 \mathrm{nT} ; R_{D 1}=92.07 R_{J} ; R_{D 2}=18.4 R_{J} ;$ $B_{D C}=2.5 \mathrm{nT}$ by comparison with the Ulysses inbound pass data (see Fig. 5) in the middle and outer magnetosphere $\left(r>15 R_{J}\right)$. The calculated magnetic field strength in the subsolar magnetopause is $B_{\mathrm{Ss}} \sim 4.69 \mathrm{nT}$. In the closed magnetospheric model the subsolar point has two polar cap projections in the noon meridian with the magnetic latitudes $\pm 84.23^{\circ}$ (for the Jovian dipole field plus the field of its screening magnetopause currents, the corresponding cusp latitudes are $\left.\pm 87.12^{\circ}\right)$. The coordinates of the magnetic field neutral points (cusps) for the chosen model parameters are: $x=55.48 R_{\mathrm{J}}, y=0, z= \pm 76.86 R_{\mathrm{J}}$. For comparison, in the Engle and Beard (1980) model deduced from Pioneer 10 magnetic field observations and using an equatorial current sheet from $\sim 17.9$ to $100 R_{\mathrm{J}}$, where the current decreases as $1 / r^{1.7}$, the subsolar point distance was equal to 100 planetary radii and the net subsolar point field was $4.534 \mathrm{nT}$. In their model the cusps could be found at: $x=26 R_{\mathrm{J}}, y=0, z= \pm 65 R_{\mathrm{J}}$.

Here we neglect the twisting of magnetic field lines by planetary rotation. An angle characterizing this twisting is estimated as $\arctan \left(2 \pi r / T_{\mathrm{J}} V_{\mathrm{A}}\right)$, where $r$ is the radius of a magnetotail lobe, $T_{\mathrm{J}}$ is the rotation period $(9.925 \mathrm{~h})$, and $V_{\mathrm{A}}$ is the local Alfvén speed in the Jovian tail lobes (Goldstein et al., 1986 and references therein). According to the observations and estimations of Goldstein et al. $(1985,1986)$, the amount of twist is only $2^{\circ}-3^{\circ}$. So, the significant twisting of the lobe field lines is not supported by the data.

Figure 8 presents projections along magnetic field lines of the constant latitude with a $2^{\circ}$-step (solid curves) and constant longitude with a 2-h step (dashed curves). As it was shown by Belenkaya $(2003,2004)$, the scalar potential of the electric field caused by Jupiter's rotation depends on the ionospheric latitude, so the projections of the constant ionospheric latitude are the electric field equipotentials.

\subsection{Dependence of the Jovian magnetosphere on the solar wind pressure}

The constructed magnetospheric model and the measurements on board the Ulysses spacecraft during its first flyby of the dayside equatorial Jovian magnetosphere are used below to formulate the pressure balance equation at the subsolar point. This equation takes into account the magnetodisc presence. The solution of this equation yields a dependence of the magnetospheric scale on the solar wind dynamic pressure. Our results are in good agreement with the magnetopause crossing data analysis by Huddlestone et al. (1998).

Observations made during the first Ulysses pass by Jupiter at the beginning of February 1992 have detected the magnetopause at distances of 110-90 $R_{J}$ (Hawkins et al., 1998). 


\section{Jovian magnetospheric field. All current systems.}

\section{Dipole, current disc, tail current, and magnetopause screening currents}

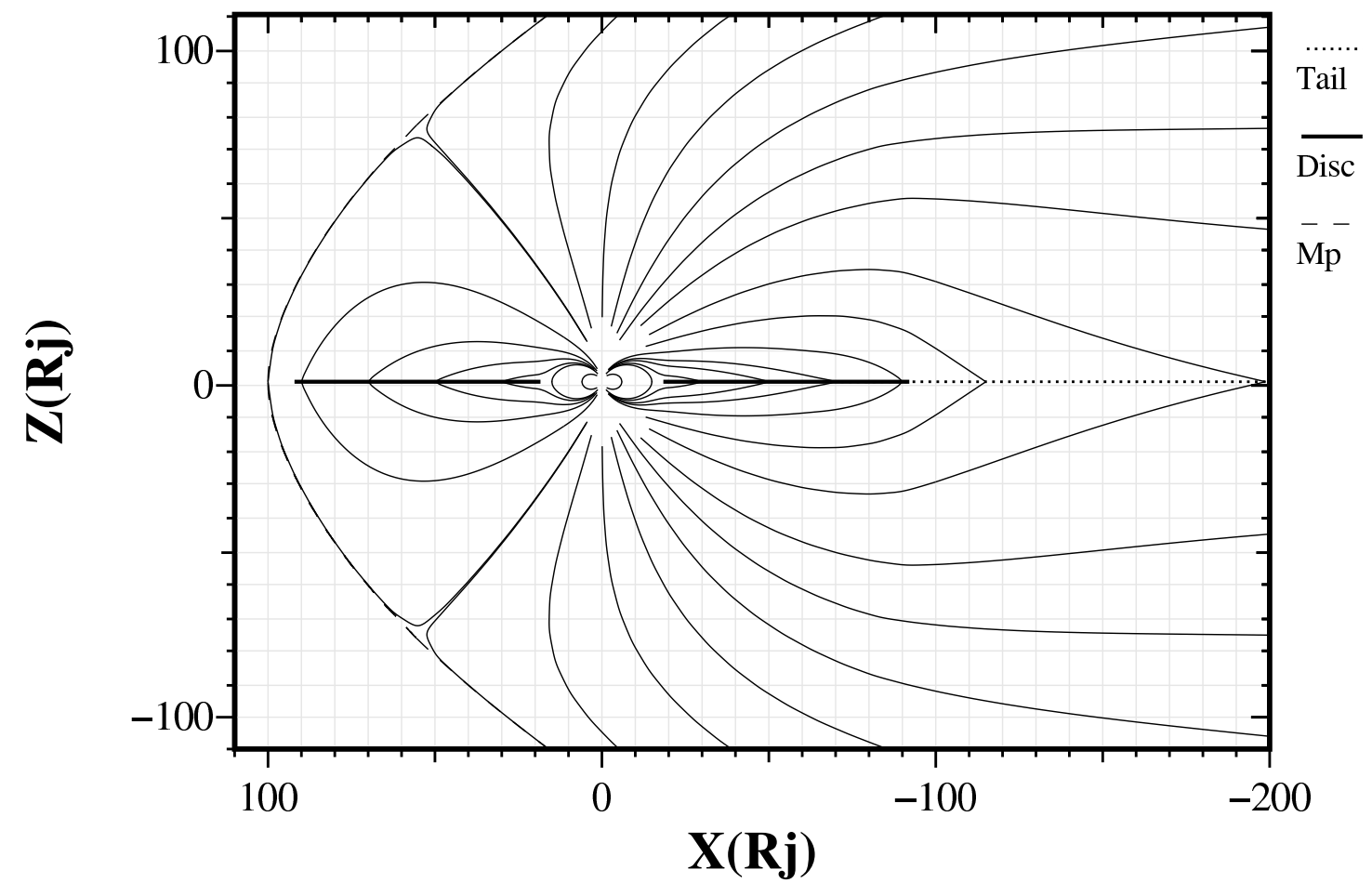

Fig. 7. Noon-midnight meridional cross section. Total magnetic field from all magnetospheric sources screened by the magnetopause currents. Dashed line marks the magnetotail current sheet. The chosen parameters: $R_{\mathrm{Ss}}=100 R_{\mathrm{J}} ; R_{\mathrm{D} 1}=92.07 R_{\mathrm{J}} ; R_{\mathrm{D} 2}=18.4 R_{\mathrm{J}} ; B_{\mathrm{DC}}=2.5 \mathrm{nT}$. Northward (southward) from the equatorial plane, the magnetic field is directed from (to) Jupiter.

The Ulysses solar wind plasma data allow one to calculate the dynamic pressure just outside the Jovian bow shock $p_{s w J}=18 \mathrm{pPa}$ from the solar wind density, $n_{s w J}=0.06 \mathrm{~cm}^{-3}$, and velocity, $V_{s w}=400 \mathrm{~km} / \mathrm{s}$ (see Figs. 9 and 10). The Ulysses spacecraft intersects the magnetopause at latitude $5^{\circ}$ at 10:30 UT, measuring the magnetospheric magnetic field $B_{m J}=4.69 \mathrm{nT}$. This magnetic field gives one half of the pressure $\left(B_{m J}^{2} / 2 \mu_{0}=8.75 \mathrm{pPa}\right)$ which is needed for the pressure balance at the subsolar point. It is reasonable to propose that the deficit of the magnetospheric pressure, $\sim 9 \cdot 10^{-12} \mathrm{~Pa}$, is provided by the magnetospheric plasma. In this case, the magnetospheric plasma pressure is about the same as the magnetospheric magnetic field pressure $\left(\sim 9 \cdot 10^{-12} \mathrm{~Pa}\right)$.

We took into account three contributors to the magnetospheric pressure at the subsolar point:

1. the dipole field together with the dipole's screening magnetopause current field,

2. the magnetodisc and its screening current fields, and

3. magnetospheric plasma pressure.

Neglecting the tail current field is valid because the model calculations show that it gives only $1 \%$ to the total field strength at subsolar point. The dipole field strength is proportional to $r^{-3}$. So, for arbitrary $p_{s w J}$ the first term of the contribution to subsolar magnetic field is $1.31 \mathrm{nT}\left(\frac{100 R_{J}}{R_{s s}}\right)^{3}$. Here $1.31 \mathrm{nT}$ is a model calculated strength of the dipole and the dipole's screening current field for $R_{s s}=100 R_{J}$.

The magnetodisc field is proportional to $r^{-2}$. In the inner magnetosphere (at $r \sim 20 R_{J}$ ) the magnetodisc current field is essential, but the other magnetospheric current fields give negligible small contributions there. We propose that the distance to the inner edge of the magnetodisc and the disc current density are not changed by the solar wind pressure forcing or weakening. According to our suggestion, the solar wind pressure and consequently the magnetospheric scale control only the outer boundary of the Jovian plasma disc. In this case, for arbitrary $p_{s w J}$, the second term in the subsolar magnetic field is $3.38 \mathrm{nT}\left(\frac{100 R_{J}}{R_{s s}}\right)^{2}$. Here $3.38 \mathrm{nT}$ is the model calculated strength of the magnetodisc and its screening current field for $R_{s s}=100 R_{J}$. If magnetospheric scale $R_{S S}$ is measured in $R_{J}$, then the total Jovian magnetospheric field pressure at the subsolar point is

$p_{B}=B_{m J}^{2} / 2 \mu_{0}=4.55 \frac{10^{8}}{R_{s s}^{4}}\left(1+\frac{77}{R_{s s}}+\frac{1494}{R_{s s}^{2}}\right) \mathrm{pPa}$.

It is more difficult to define the plasma pressure at the subsolar point, $p_{m s}$, because we do not know exactly the plasma parameters inside the plasma disc. In the latitude direction 


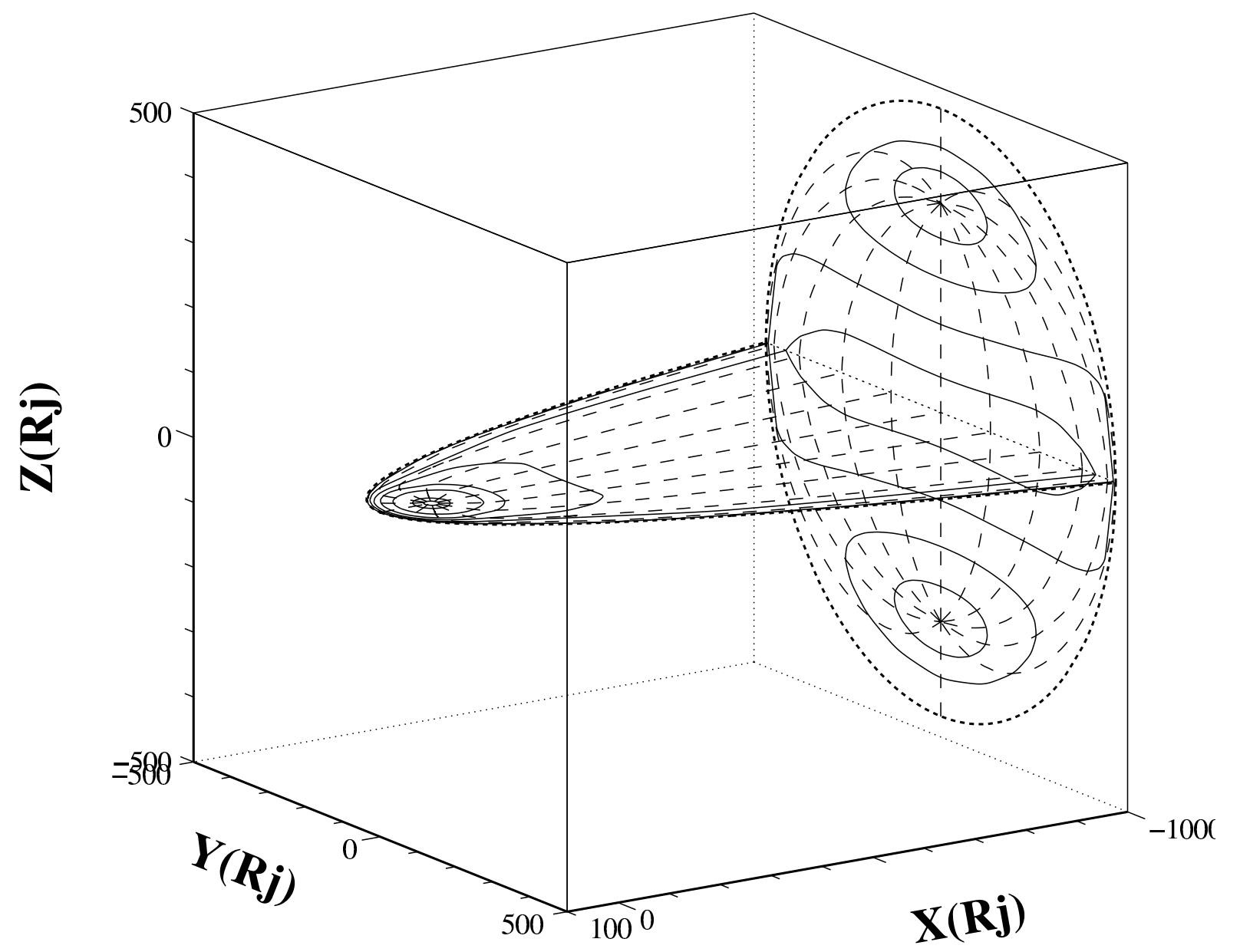

Fig. 8. Projections of the constant Jovian latitude with step $2^{\circ}$ (solid curves) and constant longitude with a 2-h step (dashed curves) along magnetic field lines. Model parameters: $\Psi=0 ; R_{\mathrm{SS}}=100 R_{\mathrm{J}} ; R_{2}=65 R_{\mathrm{J}} ; B_{\mathrm{t}}=-2.5 \mathrm{nT} ; R_{\mathrm{D} 1}=92 R_{\mathrm{J}} ; R_{\mathrm{D} 2}=18.4 R_{\mathrm{J}} ; B_{\mathrm{DC}}=2.5 \mathrm{nT}$. The constant latitude curves in the equatorial plane are from $72^{\circ}$ to $84^{\circ}$, and in the tail cross section from $82^{\circ}$ to $90^{\circ}$.

the total pressure must be constant. We propose that the plasma pressure $p_{m}$ inside the plasma disc is equal to the magnetospheric field pressure $B_{m}^{2} / 2 \mu_{0}$ outside the disc. This relation is correct at the subsolar point, too (Caudal, 1986; Caudal and Connerney, 1989). This conclusion is supported by observations made during the first Ulysses pass by Jupiter at the beginning of February 1992 (Hawkins et al., 1998).

For $R_{s s}=100 R_{J}$, the model calculations give $p_{m s}=p_{B} \simeq 8.75 \mathrm{pPa}$. For arbitrary $p_{s w J}$ a good approach for $p_{m s}$ is $p_{m s}=8.75 \frac{10^{8}}{R_{s s}^{4}} \mathrm{pPa}$, because $B_{m J} \sim r^{-2}$ is the main term of $B_{m J}$.

Finally, we write the pressure balance equation:

$p_{s w J}=0.58 p_{0 J}=13.3 \frac{10^{8}}{R_{s s}^{4}}\left(1+\frac{26.3}{R_{s s}}+\frac{511}{R_{s s}^{2}}\right) \mathrm{pPa}$.

With good accuracy the solution of Eq. (24) can be presented as

$R_{s s}=\left(\frac{39.81}{p_{s w J}^{0.23}[\mathrm{nPa}]}\right) R_{J}$.
In Fig. 11 a comparison of our solution with the results obtained by Huddlestone et al. (1998) is shown. For both axes in Fig. 11 the logarithm scales are used. The results from Huddlestone et al. (1998) give a line which is described by equation:

$R_{s s}=\frac{35.5 R_{J}}{p_{s w}^{0.22}[\mathrm{nPa}]}$.

The curve derived from the model calculations (Eq. 25) is slightly above, but still within the error bars of the results from Huddlestone et al. (1998), based on Voyager 1 and 2 data (Eq. 26). Our result is in good agreement with that of Slavin et al. (1985), who examined Pioneer and Voyager dayside data. Slavin et al. (1985) found a $p_{s w J}$ dependence to the power -0.23 for the magnetopause subsolar distance. It coincides with the slope of our $R_{S S}$ dependence on $p_{s w J}$. The average magnetospheric scale normalized to average solar wind dynamic pressure $\left(p_{s w J} \approx 0.1 \mathrm{nPa}\right)$ for all magnetopause crossing studied by Huddlestone et al. (1998) is $69.1 R_{J}$. The solution of Eq. (24) gives $67.6 R_{J}$. 


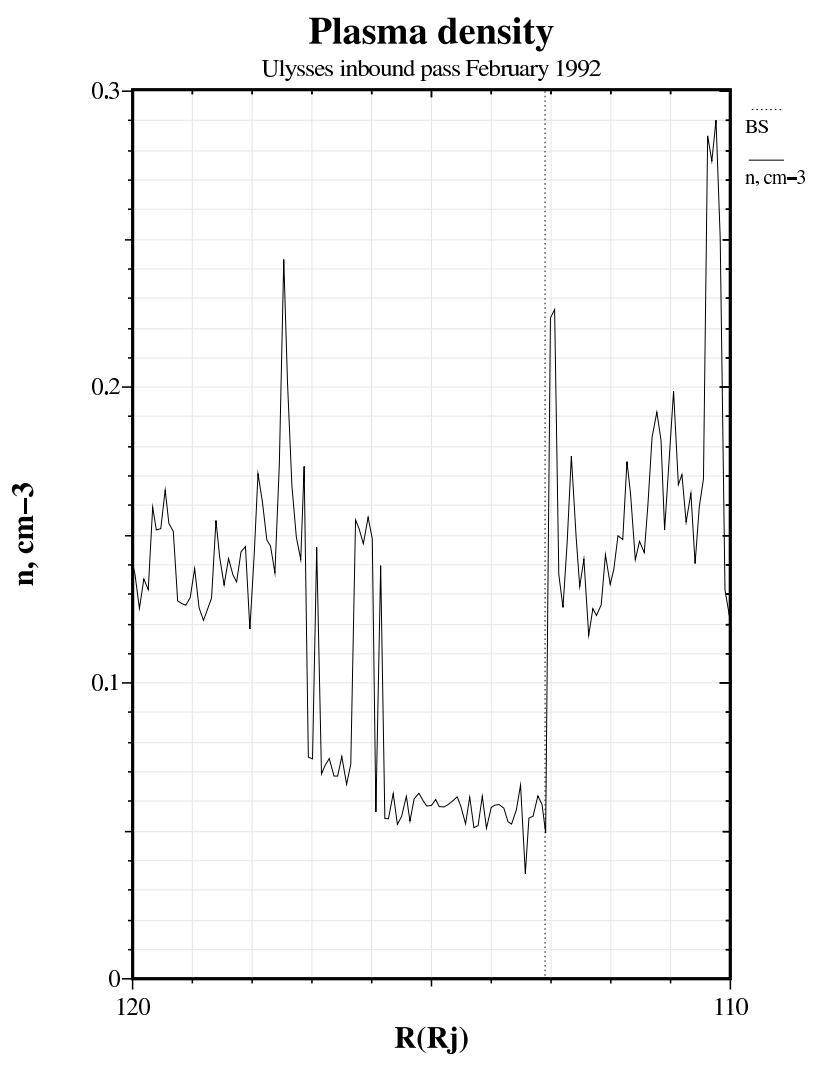

Fig. 9. Measured by SWOOPS plasma density in the course of the bow shock crossing by Ulysses during the first Jupiter flyby, February 1992. The bow shock position is marked by a dashed vertical line. Data (SWOOPS, PI J. L. Phillips LANL, USA) are received by using the COHOWEB system, NSSDC, NASA/GSFC, USA; ftp://nssdcftp.gsfc.nasa.gov/spacecraft_data/ ulysses/plasma/swoops/jupiter.

\section{Open Jovian magnetosphere}

Belenkaya $(2003,2004)$ showed that the model allows one to not only calculate the magnetospheric magnetic field everywhere in the magnetosphere, but also to take into account the interplanetary magnetic field (IMF). Including currents on the magnetopause, which shield all magnetospheric magnetic field sources, we can calculate the effects caused by the IMF penetration into the magnetosphere, in particular, the electric field created by the MHD solar wind generator.

As it was shown by Alexeev (1986), in spite of the full screening of the inner magnetospheric fields by the magnetopause currents, during the flowing of the solar wind pass, the paraboloid of revolution of the interplanetary magnetic field partially penetrates the magnetosphere. For the case of Jupiter, the ratio of the penetrated field value $(b)$ to that outside of the IMF $\left(B_{I M F}\right)$ is named $k_{J}$. The existence of the plasma flow out of the magnetosphere leads to different mechanisms of the magnetic field penetration through the boundary plasma-field (magnetopause) along two antiparallel directions perpendicular to this boundary. The solar wind

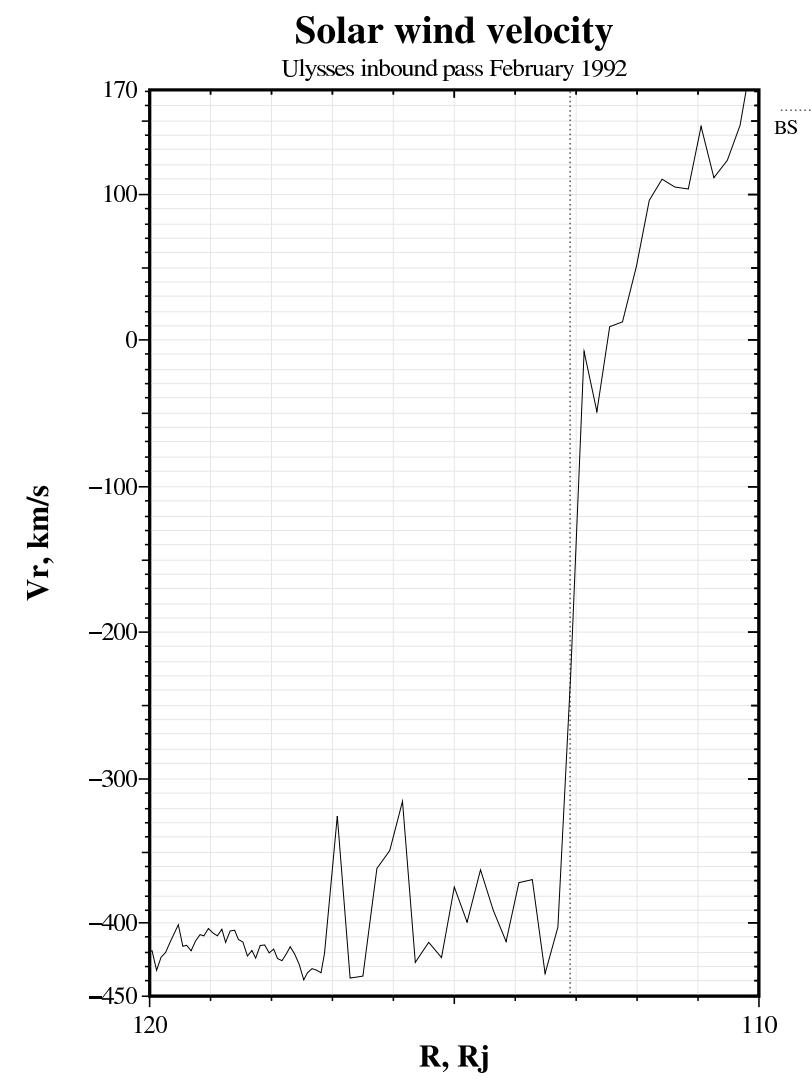

Fig. 10. Measured by SWOOPS plasma velocity in the course of the bow shock crossing by Ulysses during the first Jupiter flyby, February 1992. The bow shock position is marked by a dashed vertical line. Data (SWOOPS, PI J. L. Phillips LANL, USA) are received by using the COHOWEB system, NSSDC, NASA/GSFC, USA; ftp://nssdcftp.gsfc.nasa.gov/spacecraft_data/ ulysses/plasma/swoops/jupiter.

magnetic field partially penetrates into the magnetosphere, while the magnetospheric magnetic field drifts away by the flow in the magnetosheath. In Paper 1 it was shown that for the typical value of southward IMF $(0.5 \mathrm{nT})$ and $k_{J}$ of the order of 0.8 , the magnitudes of width of the anti-corotational layer in the equatorial noon-dawn outer Jovian magnetosphere, calculated in the presented model and measured by the Ulysses, are close to each other.

The Jovian atmosphere provides a viscous transfer of momentum from the rotating interior of the planet up into the ionosphere, where the plasma is set into corotation by the collisional friction between the ions and the neutral particles. The corotation electric field is transmitted outward into the magnetosphere by highly conducting magnetic field lines (Hill, 1979). In Jupiter's magnetosphere, corotation is generally considered to break down beyond the "Alfvén point", $L_{\mathrm{A}}$, at which $\Omega_{\mathrm{J}} L_{\mathrm{A}}=V_{\mathrm{A}}$, where $V_{\mathrm{A}}$ is the local Alfvén speed (Hill, 1979). On the other side, we assume that the interplanetary magnetic field normal component to the magnetopause and interplanetary electric field component tangential to the magnetopause penetrate into the magnetosphere 


\section{Subsolar distanse on pressure dependence}

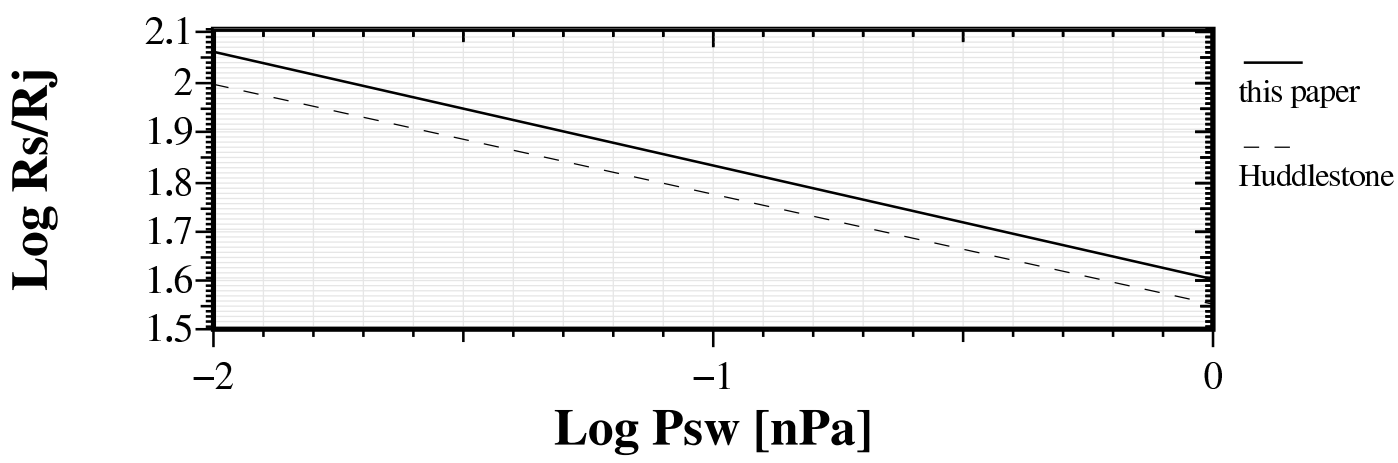

Fig. 11. Comparison of the solution of Eq. (24) (upper line) with the results by the analysis of Huddlestone et al. (1998) based on Voyager 1 and 2 data (bottom line).

with coefficient $k_{\mathrm{J}} \leq 1$. The solar wind electric potential generated at the Jovian magnetopause can be mapped along equipotential magnetic field lines into the magnetosphere up to the ionosphere along the highly conducting magnetic field lines. It was shown (see Paper 1) that the IMF redistributes rotation momentum in the Jovian magnetosphere.

Figures 12 and 13 (see also Paper 1) show electric equipotentials for the above model for the southward and northward IMF, respectively. Solid curves mark equipotentials caused by the planet's rotation, and dash-dotted curves are the equipotentials of the solar wind electric field penetrated into magnetosphere. It was supposed that the IMF (with a value of $\sim 0.5-1 \mathrm{nT}$ ) penetrates into magnetosphere with a coefficient of $k_{\mathrm{J}} \sim 0.8$.

As is seen from Figs. 12 and 13, behind the region of corotation in the equatorial plane, the antisunward flow takes place independent of the sign of $B_{z}$ IMF. Taking into account the observed corotation braking near the equatorial plane, in Paper 1 it was obtained that in the noon outer low-latitude Jovian magnetosphere, the anti-corotational and antisunward flows exist. Such flows were observed by Ulysses entering Jupiter's magnetosphere when IMF $B_{z}$ was southward (e.g. Staines at al., 1993). Contrary to the case of southward IMF, for northward solar wind magnetic field, corotation exists in the low-latitude Jovian magnetosphere out to the dayside magnetopause, and beyond the neutral line a nightside outflow takes place in accordance with the Pioneer 10 and Voyagers measurements (Krimigis et al., 1979; Cheng and Krimigis, 1989; Kane et al., 1992).

Meanwhile, in Figs. 14 at the scheme of the thermal plasma flows in the Jovian and terrestrial magnetospheres Brice and Ioannidis (1970) proposed that beyond corotation in Jupiter's tail the sunward motion exists. This scheme of the convection in the Jovian magnetosphere was suggested to be analogous with the case of the Earth in the time when insitu observations were absent. However, during the Ulysses, Pioneer and Voyager measurements the antisunward motions in the tail were measured.
Cheng and Krimigis (1989) proposed a global model of plasma convection in Jupiter's equatorial magnetosphere described by an electric potential

$U=k_{1}(r \sin \theta)^{-1}+k_{2} y$

(Brice and Ioannidis, 1970), where $r \sin \theta$ is a radius in the cylindrical coordinate system, and $Y$ is directed to dusk ( $r$ and $y$ are measured in $R_{\mathrm{J}}$ ). Here $k_{1}=\Omega_{\mathrm{J}} B_{0 \mathrm{~J}} R_{\mathrm{J}}^{2}$, where $B_{0 \mathrm{~J}}=4.2 \cdot 10^{5} \mathrm{nT}$ is the magnetic field at Jupiter's equator; $\Omega_{\mathrm{J}} \approx 1.76 \cdot 10^{-4} \mathrm{~s}^{-1}$ is an angular velocity of Jupiter's rotation. The value of $k_{1}$ is equal to $k_{1}=\Omega_{\mathrm{J}} B_{0 \mathrm{~J}} R_{\mathrm{J}}^{2} \approx 377 \mathrm{MV}$. Function $k_{2}$ is determined as $k_{2}=k_{\mathrm{J}} V_{\mathrm{SW}} B_{\mathrm{IMF}} R_{\mathrm{J}}$, where $k_{\mathrm{J}}$ is a coefficient of IMF penetration, $V_{\mathrm{SW}} \approx 420 \mathrm{~km} / \mathrm{s}$ is the solar wind velocity, and $B_{\mathrm{IMF}} \approx 1 \mathrm{nT}$ is the IMF value. The magnitude of $k_{2} \approx 3 \mathrm{kV}$ for $k_{\mathrm{J}} \approx 0.1$ is given, as well as of $k_{2} \approx 30 \mathrm{kV}$ for $k_{\mathrm{J}} \approx 1$. Equation $U=k_{1} \rho^{-1}+k_{2} y=0$ is fulfilled for southward IMF at $y=-354 R_{\mathrm{J}}$ for $k_{\mathrm{J}} \approx 0.1$ and at $y=-112 R_{\mathrm{J}}$ for $k_{\mathrm{J}} \approx 1$. Thus, according to Eq. (27) of Cheng and Krimigis (1989), using the presentation of the electric potential by Brice and Ioannidis (1970), the corotation field is equal and antiparallel to the field generated by the solar wind in the dawn side far out of the magnetosphere for $k_{\mathrm{J}} \approx 0.1$, and probably inside the magnetosphere, near the magnetopause, for $k_{\mathrm{J}} \approx 1$.

So, the values of convection and corotation potentials with different signs (for $B_{z}<0$ ) become comparable in the dawn sector of the magnetosphere only for the relatively large $k_{\mathrm{J}} \approx 1$. From Fig. 14, however, it follows that the corotation and convection plasma flows are parallel to each other in the morning, which is not supported by observations and our calculations (see Paper 1 and references therein). Thus, the proposed model of the Jovian magnetospheric magnetic field allows one to correct the ideas about the influence of the solar wind electric field on Jupiter's environment. 


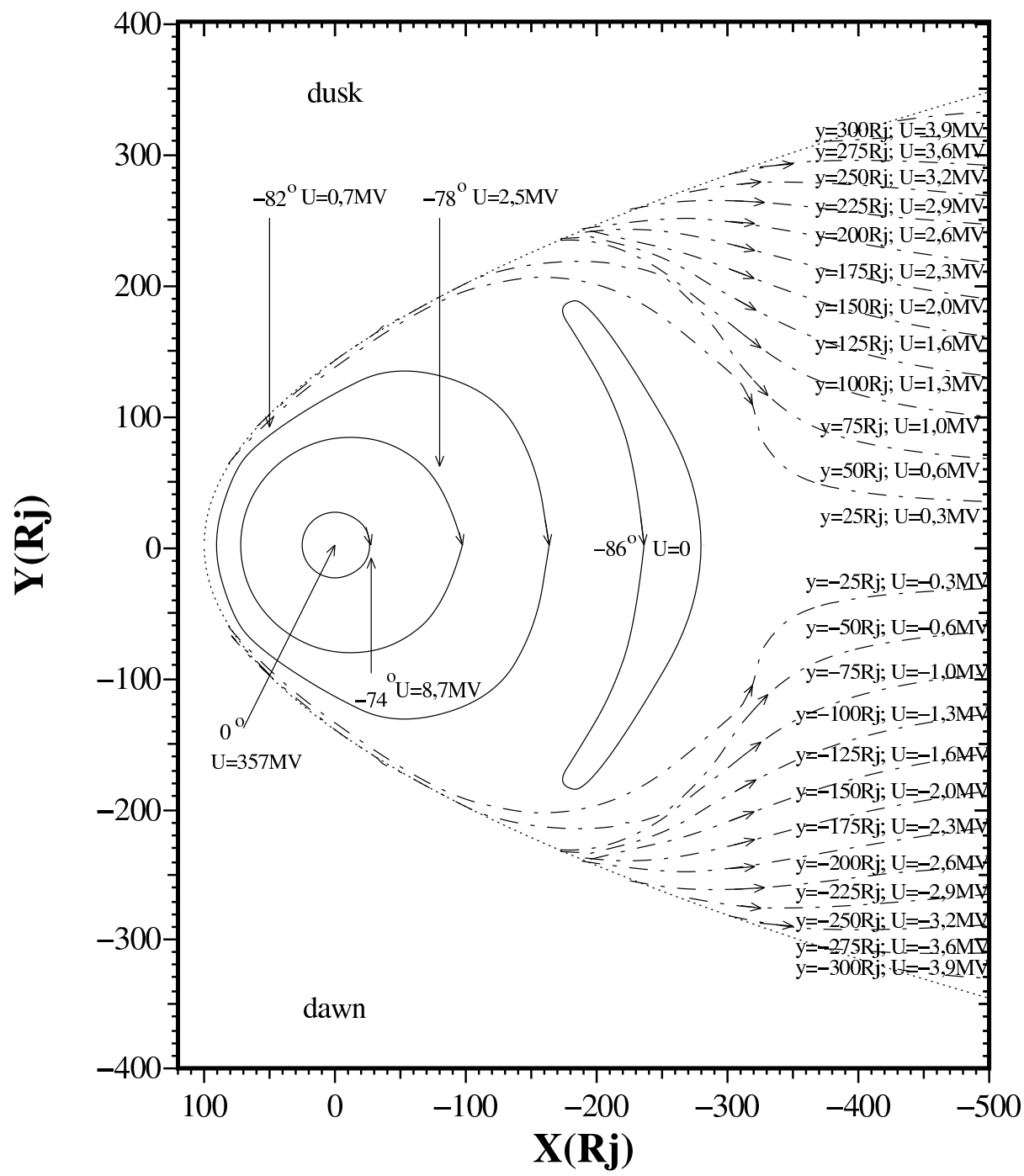

Fig. 12. Equatorial projection (solid curves) of lines of constant ionospheric latitude (the corotation electric equipotentials). The dash-dotted curves are the equatorial projections of the solar wind electric equipotentials $y=$ const with steps $\delta y=25 R_{\mathrm{J}} ; \delta U=0.3 \mathrm{MV}$. The dotted curve marks the magnetopause.

\section{Conclusions}

A model which allows us to investigate the IMF and solar wind influence on Jupiter's magnetosphere is constructed. The main effort was directed at the construction of the dynamic model of the Jovian magnetosphere. The magnetospheric dimension scale (magnetopause subsolar distance $R_{s S}$ ) depends on the solar wind dynamic pressure $p_{s w J}$ to the power -0.23 . This law is derived by us as a solution of the balance equation at the subsolar point. It coincides with the previous findings by Slavin et al. (1985) and Huddleston et al. (1998). A comparison of the presented magnetic field model with the Ulysses magnetometer data during its first Jupiter flyby shows a good agreement.

Solar wind dynamic pressure at the magnetopause crossing time can be estimated by using plasma data upstream of the bow shock. This estimation gives the pressure value
$18 \mathrm{pPa}$. Substracting from it the measured magnetospheric magnetic field pressure $8.75 \mathrm{pPa}$ shows that the magnetospheric plasma pressure is equal to the magnetic pressure at the subsolar point.

The magnetospheric dimension scale measured by Ulysses is about twice comparing to dipole magnetosphere (Earth's type). Such increasing of the subsolar distance is caused by the magnetodisc forming. The magnetodisc plasma pushes out the magnetic field from the disc region and cancels normal to the disc surface magnetic field component. It transports magnetic flux from the inner magnetosphere to the magnetopause and changes the dipole magnetic field dependence $r^{-3}$ to a slower function $r^{-2}$. An effective dipole magnetic moment is bigger than the Jovian dipole moment by $\sim 2.6$ times. The centrifugal force of the magnetodisc plasma generated by Io results in the fact that just at the magnetopause the plasma energy approximately equals the 


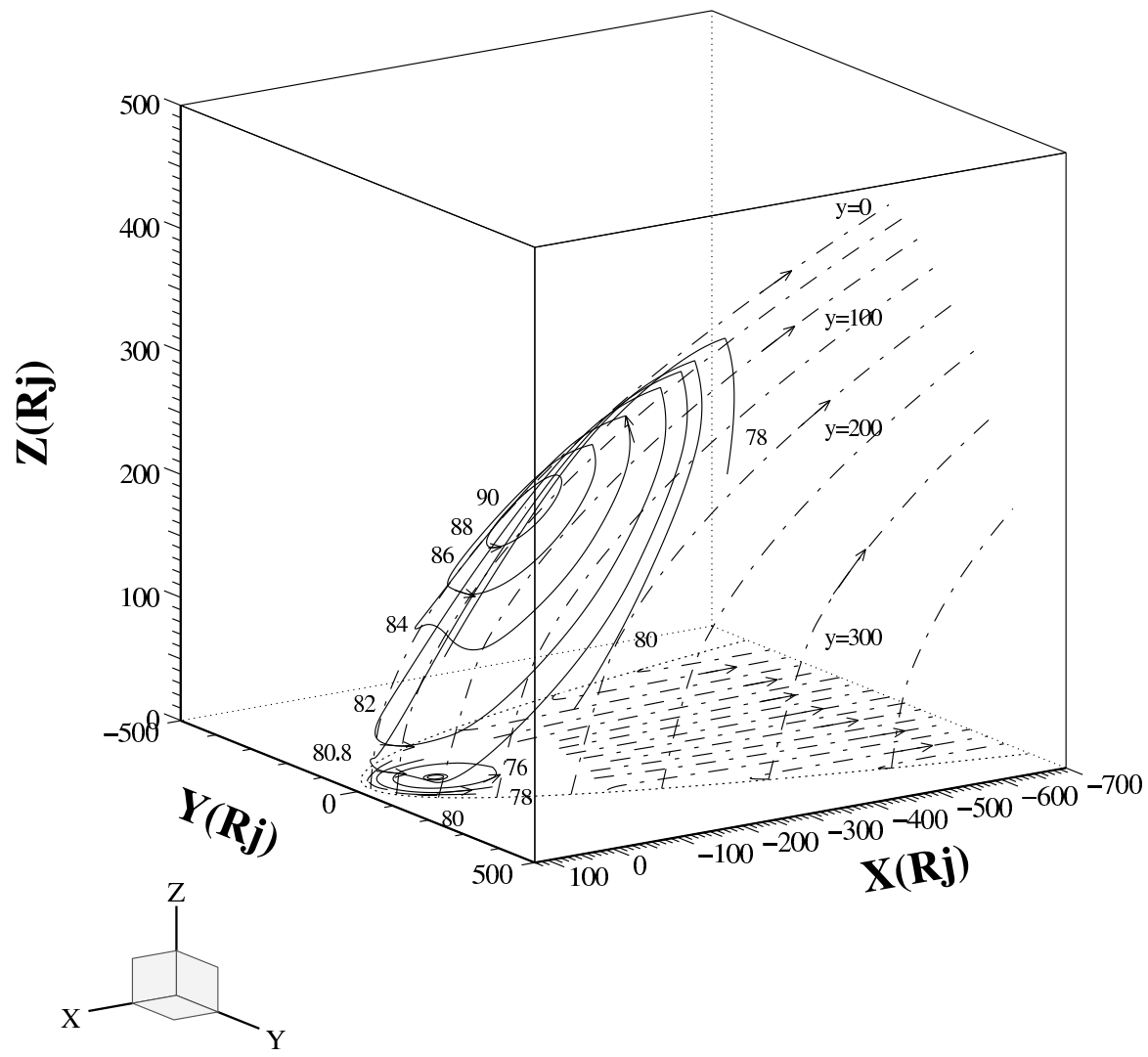

Fig. 13. Three-dimensional Jovian magnetosphere for northward IMF. Equipotentials of the corotation (solid curves) and of the solar wind (dash-dotted curves) electric field on the magnetopause and on the equatorial plane. The IMF components are: $B_{0 \mathrm{x}}=-0.02 \mathrm{nT}, B_{0 \mathrm{y}}=0$, $B_{0 \mathrm{z}}=0.5 \mathrm{nT}$; the coefficient of IMF penetration is $k_{\mathrm{J}}=1$. For the dash-dotted curves, $\delta y=50 R_{\mathrm{J}}$ and $\delta U=0.75 \mathrm{MV}$. For the solid curves on the equatorial plane (from the outer to inner) the latitudes and electric corotation potentials are: $80^{\circ}, 0.4 \mathrm{MV} ; 78^{\circ}, 1.4 \mathrm{MV} ; 76^{\circ}, 4.3 \mathrm{MV} ; 74^{\circ}$, $7.6 \mathrm{MV} ; 0^{\circ}, 356 \mathrm{MV}$, respectively. For the solid curves on the magnetopause (from the outer to inner) the corresponding values are: $81^{\circ}$, $0 \mathrm{MV} ; 82^{\circ},-1.9 \mathrm{MV} ; 84^{\circ},-5.1 \mathrm{MV} ; 86^{\circ},-7.4 \mathrm{MV} ; 88^{\circ},-8.8 \mathrm{MV} ; 90^{\circ},-9.2 \mathrm{MV}$, respectively.
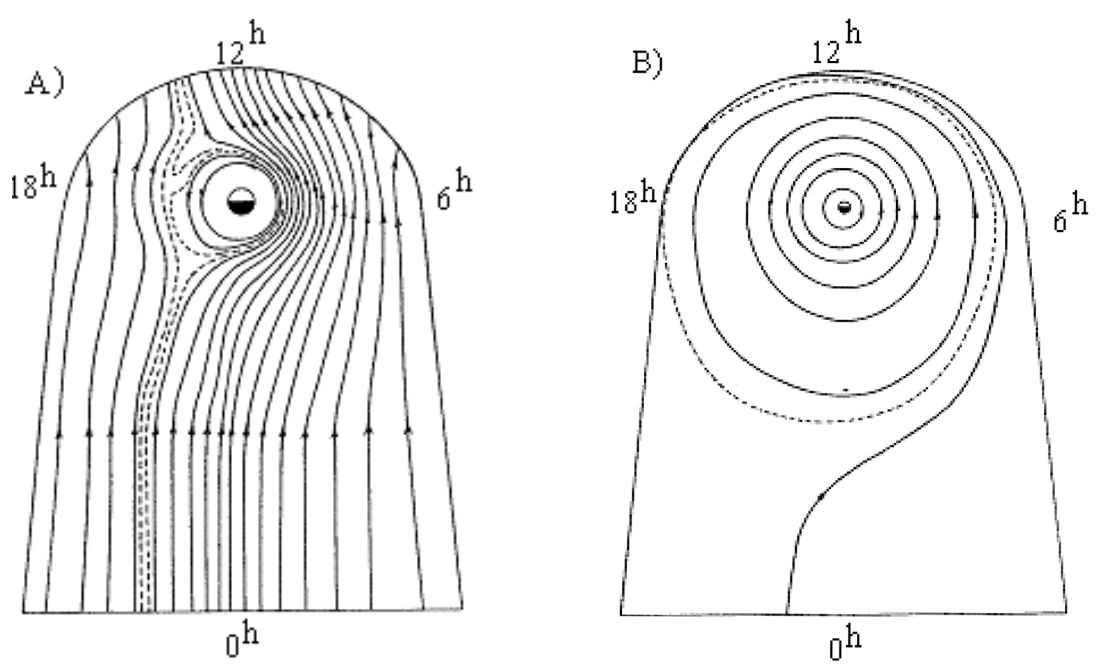

Fig. 14. Equatorial sections of the terrestrial and Jovian magnetospheres. Thermal plasma flows are shown by arrows. (A) Earth's magnetosphere; dashed curve marks the boundary between the corotation and sunward motion. (B) Jupiter's magnetosphere; dashed circle is a suggested boundary between the corotation and sunward motions (Brice and Ioannidis, 1970). 
magnetic field energy (in agreement with the results by Caudal, 1986; Caudal and Connerney, 1989).

We demonstrate the model results for a dipole tilt angle equal to zero, but the model expressions give us a possibility to calculate the magnetospheric field for an arbitrary tilt angle. We parameterized the model by the Ulysses flyby data, but in the future plan to repeat the same procedure for other available spacecraft magnetic data. For the planetary inner magnetic field we used a dipole term. However, for calculations near the planet, it is better to use the O6 (Connerney, 1993) or any other more precise inner source model. For simplicity we did not use the refined-shaped magnetodisc model and FAC field. In the vicinity of the real magnetodisc, it can give a non correct magnetic field strength, as one can see in Fig. 5.

A knowledge of the magnetospheric dimension scale dependence on the solar wind dynamic pressure allows us to find the model parameters for arbitrary solar wind pressure. It gives a possibility to study in the future the Jovian magnetospheric response to the solar wind shock or other type of solar wind pressure jump.

Acknowledgements. This work was partly supported by the RFBR Grant 04-05-64396 and by the INTAS Grant No 03-51-3922. Authors thank N. Papitashvili, COHOWEB system, NSSDC, NASA/GSFC, USA; PI A. Balogh, Imperial College, London, UK, and PI J. L. Phillips, LANL, USA for providing magnetometer and plasma (SWOOPS) Ulysses data which were received by by using COHOWEB system, NSSDC, NASA/GSFC, USA (ftp://nssdcftp.gsfc.nasa.gov/ spacecraft_data/ulysses/plasma/swoops/jupiter and ftp://nssdcftp. gsfc.nasa.gov/spacecraft_data/ulysses/mag/jupiter/minute).

Topical Editor T. Pulkkinen thanks three referees for their help in evaluating this paper.

\section{References}

Acuña, M. H., Behannon, K. W., and Connerney, J. E. P.: Jupiter's magnetic field and magnetosphere, in: Physics of the Jovian Magnetosphere, Cambridge, Plan. Sci. ser, edited by: Dessler, A. J., Cambridge, Univ. Press, New York, 1-50, 1983.

Alexeev, I. I.: Jovian magnetosphere, Itogi Nauki i Techniki, seria Issledovanie kosmicheskogo prosytanstva (in Russian), 7, VINITI, Moscow, 154-220, 1976.

Alexeev, I. I.: The penetration of interplanetary magnetic and electric fields into the magnetosphere, J. Geomag. Geoelectr., 38, 1199-1221, 1986.

Alexeev, I. I. and Shabansky, V. P.: A model of a magnetic field in the geomagnetosphere, Planet. Space Sci., 20, 117, 1972.

Alexeev, I. I., Belenkaya, E. S., and Clauer, C. R.: A model of region 1 field-aligned currents dependent on ionospheric conductivity and solar wind parameters, J. Geophys. Res., 105, 21 11921 127, 2000.

Barbosa, D. D., Gurnett, D. A., Kurth, W. S., and Scarf, F. L.: Structure and properties of Jupiter's magnetoplasmadisc, Geophys. Res. Lett., 6, No 10, 785-788, 1979.

Barish, F. D. and Smith, R. A.: An analytical model of the Jovian magnetosphere shape, Geophys. Res. Lett., 2, 269-272, 1975.

Baron, R. L. and Owen, C. J.: Solar wind control of Jupiter's $H_{3}^{+}$ auroras, Icarus, 120, No 2, 437-442, 1996.
Beard, D. B. and Jackson, D. J.: The Jovian magnetic field and magnetosphere, J. Geophys. Res., 81, 3399-3401, 1976.

Behannon, K. W., Burlaga, L. F., and Ness, N. F.: The Jovian magnetotail and its current sheet, J. Geophys. Res., 86, 8385-8401, 1981.

Belenkaya, E. S.: Peculiarities of the interaction between the solar wind magnetic field with the terrestrial and Jovian magnetospheres, Geomagn. and Aeronom (in Russian), 43, No 2, 174 182, 2003.

Belenkaya, E. S.: The Jovian magnetospheric magnetic and electric fields: effects of the interplanetary magnetic field, Planet. Space Sci., 52, 499-511, 2004.

Bespalov, P. A. and Davidenko, S. S.: On the structure, of the plasma disc in the Jovian magnetosphere, Planet. Space Sci., 42 No 7, 583-592, 1994.

Brice, N. and Ioannidis, G.: The magnetospheres of Jupiter and Earth, Icarus, 13, 173-183, 1970.

Bunce, E. J., and Cowley, S. W. H.: Local time asymmetry of the equatorial current sheet in Jupiter's magnetosphere, Planet. Space Sci., 49, 261-274, 2001.

Caudal, G.: A self-consistent model of Jupiter's magnetodisc including the effects of centrifugal force and pressure, J. Geophys. Res., 91, 4201-4221, 1986.

Caudal, G. and Connerney, J. E. P.: Plasma pressure in the environment of Jupiter, inferred from Voyager magnetometer observation, J. Geophys. Res., 94, 15 055-15 061, 1989.

Cheng, A. F. and Krimigis, S. M.: A model of global convection in Jupiter's magnetosphere, J. Geophys. Res., 94, No A9, 12003 $12008,1989$.

Connerney, J. E. P., Acuña, M. H., and Ness, N. F.: Modeling the Jovian current sheet and inner magnetosphere, J. Geophys. Res., 86, 8370-8384, 1981.

Connerney, J. E. P.: Magnetic fied of the uter planets, J. Geophys Res., 98, 18659-18679, 1993.

Connerney, J. E. P., Satoh, T., and Baron, R. L.: Interpretation of auroral lightcurves with application to Jovian $\mathrm{H}_{3}^{+}$emissions, Icarus, 122, No 1, 24-35, 1996.

Connerney, J. E. P., Acuña, M. H., Ness, N. F., and Satoh, T.: New models of Jupiter's magnetic field constrained by the Io flux tube footprint, J. Geophys. Res., 103, N A6, 11 929-11 940, 1998.

Cowley, S. W. H.: Asymmetry effects associated with the $\mathrm{x}$ component of the IMF as in a magnetically open magnetosphere, Planet. Space Sci., 29, N 8, 808-818, 1981.

Cowley, S. W. H. and Bunce, E. J.: Modulation of Jupiter's main auroral oval emissions by solar wind induced expansions and compressions of the magnetosphere, Planet. Space Sci., 51, 57-79, 2003.

Cowley, S. W. H., Balosh, A., Dougherty, M. K., Dunlop, M. W., Edwards, T. M., Forbysh, R. J., Hynds, R. J., Laxton, N. F., and Staines, K.: Plasma flow in the Jovian magnetosphere and related magnetic effects: Ulysses observations, J. Geophys. Res., 101, A7, 15 197-15 210, 1996.

Cowley, S. W. H., Bunce, E. J., and Nichols, J. D.: Origins of Jupiter's main oval auroral emissions, J. Geophys. Res., 108, A4, 8002, doi:10.1029/2002JA009329, 2003a.

Cowley, S. W. H., Bunce, E. J., Stallard, T. S., and Miller, S.: Jupiter's polar ionospheric flows: Theoretical interpretation, Geophys. Res. Letts, 30, No 5, 1220, doi:10.1029/2002GL016030, 2003b.

Engle, I. M. and Beard, D. B.: Idealized Jovian magnetosphere shape and field, J. Geophys. Res., 85, 579-592, 1980.

Engle, I. M.: Idealized Voyager Jovian magnetosphere shape and 
field, J. Geophys. Res., 96, 7793-7802, 1991.

Goertz, C. K.: Jupiter's magnetosphere: Particles and fields, in: Jupiter the Giant Planet, edited by: Gehrels, T., University of Arizona Press, Tucson, Arizona, 23-58, 1976a.

Goertz, C. K.: The current sheet in Jupiter's magnetosphere, J. Geophys. Res., 81, 3368-3372, 1976b.

Goertz, C. K.: The Jovian magnetodisc, Space Sci. Rev., 23, 319343,1979

Goertz, C. K., Jones, D. E., Randall, B. A., Smith, E. J., and Thomsen, M. F.: Evidence for open field lines in Jupiter's magnetosphere, J. Geophys. Res., 81, 3393-3398, 1976.

Goldstein, M. L., Lepping, R. P., and Sittler Jr., A. C.: Magnetic field properties of Jupiter's tail at distances from 80 to 7500 Jovian radii, J. Geophys. Res., 90, 8223-8239, 1985.

Goldstein, M. L., Lepping, R. P., and Sittler Jr., A. C.: Reply, J. Geophys. Res., 91, 7133, 1986.

Greene, J. M. and Miller, R. L.: The field of a screened magnetic dipole, Planet. Space Sci., 42, No 10, 895-900, 1994.

Gurnett, D. A., Kurth, W. S., Hospodarsky, G. B., Persoon, A. M., Zarka, P., Lecacheux, A., Bolton, S. J., Desch, M. D., Farrell, W. M., Kaiser, M. L., Ladreiter, H.-P., Rucker, H. O., Galopeau, P., Louarn, P., Young, D. T., Pryor, W. R., and Dougherty, M. K.: Control of Jupiter's radio emission and aurorae by the solar wind, Nature, 415/28, 985-987, 2002.

Hawkins, S. E., Cheng, A. F., and Lanzerotti, L. J.: Bulk flows of hot plasma in the Jovian magnetosphere: A model of anisotropic fluxes of energetic ions, J. Geophys. Res., 103, 20 031-20 054, 1998.

Hill, T. W.: Inertial limit of corotation, J. Geophys. Res., 84, No A11, 6554-6558, 1979.

Hill, T. W.: The Jovian auroral oval, J. Geophys. Res., 106, 81018107, 2001.

Hill, T. W., Dessler, A. J., and Michel, F. C.: Configuration of the Jovian magnetosphere, Geophys. Res. Lett., 1, 3-6, 1974.

Huddleston, D. E., Russell, C. T., Kivelson, M. G., Khurana, K. K., and Bennett, L.: Location and shape of the Jovian magnetopause and bow shock, J. Geophys. Res., 103, 20 075-20 082, 1998.

Joy, S. P., Kivelson, M. G., Walker, R. J., Khurana, K. K., Russell, C. T., and Ogino, T.: Probabilistic models of the Jovian magnetopause and bow shock locations, J. Geophys. Res., 107, No A10, 1309, doi:10.1029/2001JA009146, 2002.

Kane, M., Mauk, B. H., Keath, E. P., and Krimigis, S. M.: A convected kappa distribution model for not ions in the Jovian magnetodisc, Geophys. Res. Lett., 19, 1435-1439, 1992.

Khurana, K. K.: Euler potential models of Jupiter's magnetospheric field, J. Geophys. Res., 102, 11 295-11 306, 1997.
Khurana, K. K.: Influence of solar wind on Jupiter's magnetosphere deduced from currents in the equatorial plane, J. Geophys. Res., 106, No A11, 25 999-26 016, 2001.

Kivelson, M. G. and Southwood, D. J.: First evidence of IMF control of Jovian magnetospheric boundary locations: Cassini and Galileo magnetic field measurements compared, Planet. Space Sci., 51, 891-898, 2003.

Krimigis, S. M., Armstrong, T. P., Axford, W. I., Bostrom, C. O., Fan, C. Y., Gloeckler, G., Lanzerotti, L. J., Keath, E. P., Zwickl, R. D., Carbary, J. F., and Hamilton, D. C.: Hot plasma environment at Jupiter: Voyager 2 results, Science, 206, 977-984, 1979.

Kurth, W. S., Gurnett, D. A., Gospodarsky, G. B., Farrell, W. M., Roux, A., Dougherty, M. K., Joy, S. P., Kivelson, M. G., R. J. Walker, R. J., Crary, F. J., and Alexander, C. J.: The dusk flank of Jupiter's magnetosphere, Nature, 415, 991-994, 2002.

Ness, N. F., Acuña, M. H., Lepping, R. P., Burlaga, L. F., Behannon, K. W., and Neubauer, F. M.: Magnetic field studies at Jupiter by Voyager 1: Preliminary results, Science, 204, 982-986, 1979.

Pontius Jr., D. H.: Radial mass transport and rotation dynamics, J. Geophys. Res., 102, 7137-7150, 1997.

Prange, R., Chagnon, G., Kivelson, M. G., Livengood, T. A., and Kurth, W. S.: Temporal monitoring of Jupiter's auroral activity with IUE during the Galileo mission. Implications for magnetospheric processes, Planet. Space Sci., 49, 405-415, 2001.

Slavin, J. A., Smith, E. J., Spreiter, J. R., and Stahara, S. S.: Solar wind flow about the outer planets: Gas dynamic modeling of the Jupiter and Saturn bow shocks, J. Geophys. Res., 90, 6275-6283, 1985.

Smith, E. J. and Wenzel K.-P.: Introduction to the Ulysses encounter with Jupiter, J. Geophys. Res., 98, 21 111-21 127, 1993.

Smith, E. J., Davis Jr., L., Jones, D. E., Coleman Jr., P. J., Colburn, D. S., Dyal, P., Sonett, C. P, and Frandsen, A. M. A.: The planetary magnetic field and magnetosphere of Jupiter: Pioneer 10, J. Geophys. Res., 79, 3501-3512, 1974.

Smith, E. J., Davis Jr., L., Jones, D. E., Coleman Jr., P. J., Colburn, D. S., Dyal, P., Sonett, C. P.: Jupiter's magnetic field, magnetosphere, and interaction with the solar wind: Pioneer 11, Science, 188, 451-454, 1975.

Spreiter, J. R., Summers, A. L., and Alksne, A. Y.: Hydromagnetic flow around the magnetosphere, Planet. Space Sci., 14, No 3, 223-253, 1966.

Staines, K., Balogh, A., Cowley, S. W. H., Edwards, T. M., Forsyth, R. J., and Hynds, R. J.: Ulysses observations of noncorotational flows in the outer dayside Jovian magnetosphere, Planet. Space Sci., 41, No 11/12, 931-946, 1993.

Walker, R. J., Ogino, T., and Kivelson, M. G.: Magnetohydrodynamic simulations of the effects of the solar wind on the Jovian magnetosphere, Planet. Space Sci., 49, 237-245, 2001. 